Supporting Information

\title{
Crystallization of Covalent Triazine Frameworks via \\ Heterogeneous Nucleation Approach for Efficient Photocatalytic Applications
}

Liping Guo, ${ }^{\text {aa }}$ Xuepeng Wang, ${ }^{\text {a }}$ Zhen Zhan, ${ }^{\mathrm{a}}$ Yueli Zhao, ${ }^{\mathrm{a}}$ Linjiang Chen, ${ }^{\text {bb }}$ Tao Liu, Bien Tan ${ }^{* a}$ and Shangbin Jin*a

a School of Chemistry and Chemical Engineering, Huazhong University of Science and Technology, Luoyu Road. 1037, Wuhan, 430074, China.

${ }^{\mathrm{b}}$ Department of Chemistry and Materials Innovation Factory \& Leverhulme Research Centre for Functional Mate-rials Design, University of Liverpool, 51 Oxford Street, Liverpool L73NY, UK. 
1. Materials and measurements

2. Synthesis of CTF-HUST-Sx with heterogeneous nucleation agent

3. Evidences for proposed mechanism

4. PXRD patterns of CTF-HUST-S2 synthesized in different nucleation agents.

5. PXRD simulation of the crystalline structures

6. HR-TEM images of CTF-HUST-Sx

7. FT-IR spectra of CTF-HUST-Sx

8. XPS of CTF-HUST-Sx

9. Thermal stability of CTF-HUST-Sx

10. Morphology of CTF-HUST-Sx

11. Dispersability of CTF-HUST-Sx

12. UV-visible absorption and optical band gaps of CTF-HUST-Sx

13. Energy level alignments of CTF-HUST-Sx

14. Photoluminescence and electrochemical performance of CTF-HUST-Sx

15. Photocatalytic hydrogen production performance of CTF-HUST-Sx

16. Elemental analysis of CTF-HUST-Sx and CTF-HUST-2 and CTF-HUST-3

17. Surface area and porosity of CTF-HUST-Sx

18. Comparison of the photocatalytic performance of CTF-HUST-Sx samples with reported results

19. Computational details

20. References 


\section{Materials and measurements}

Materials. Terephthalaldehyde, 4,4'-Biphenyldicarboxaldehyde terephthalonitrile, Tris(4formylphenyl)amine, lithium bis(trimethylsilyl)amide, dimethyl sulfoxide (DMSO), anhydrous tetrahydrofuran (THF), acetyl chloride, $\mathrm{NaCl}, \mathrm{KCl}, \mathrm{LiCl}, \mathrm{Na}_{2} \mathrm{SO}_{4}$ and $\mathrm{NaBr}$ were purchased from Aladdin. [2,2'-Bipyridine]-5,5'-dicarboxaldehyde, thieno[3,2-b] thiophene-2,5dicarboxaldehyde and 4-[3,5-bis(4-formylphenyl)phenyl]-benzaldehyde were purchased from Shanghai ChemSoon Chemical Technology Co., Ltd. Sodium borohydride, triethanolamine (TEOA), chloroplatinic acid, methanol and ethanol were purchased from Sinopharm Chemical Reagent Co., Ltd. Terephthalamidine dihydrochloride was synthesized following the reported method $^{[1]}$. All these chemicals were directly used without going through further purification.

Characterizations. The structures of the resulting crystalline CTFs were confirmed by a series of general characterization techniques, including Fourier-transformed infrared (FT-IR) spectra (ALPHA, Bruker, using ATR model), X-ray photoelectron spectroscopy (XPS) analysis (ESCALAB 250xi, Thermo Scientific) and solid-state ${ }^{13} \mathrm{C}$ NMR experiments (Avance II $600 \mathrm{MHz}$, Bruker). To reveal the porous structures, the porous properties of these crystalline CTFs were clarified by nitrogen adsorption and desorption isotherms measured at $77 \mathrm{~K}$ using liquid nitrogen (ASAP 2040, Micromeritics). The thermal stabilities of the crystalline CTFs were measured by thermogravimetric analysis (TGA) (Pyris1, PerkinElmer Instruments) with a temperature interval between 25 and $800{ }^{\circ} \mathrm{C}$. Inductively coupled plasma (ICP) spectrometer was adopted to detect the possible remaining amount of $\mathrm{NaCl}$ in the final CTFs and the cation content in real sea water. The crystalline structures of CTFs were clearly characterized by powder X-ray diffraction (PXRD) (PANalytical B.V. Empyrean). The nanosheets morphologies of these crystalline CTFs were revealed by both transmission electron microscopy (TEM) (Talos F200X, FEI) and field emission scanning electron microscope (FESEM) (NanoSEM 450, Nova) and atomic force microscopy (AFM, SPM9700, Shimadzu). The CTFs were loaded on glass plate for SEM testing. The light absorption ability of CTFs were studied by using UV-Visible diffuse reflectance spectra (DRS) (UV-3000, Shimadzu) in the solid state with a integrating sphere mode. The steady photoluminescence spectra were 
recorded using a FLS-960 fluorescence spectrometer under $365 \mathrm{~nm}$ excitation. The testing sample was dispersed in DMF with the concentration of $0.5 \mathrm{mg} \mathrm{mL}$. Electrochemical properties of the crystalline CTFs were measured using a three-electrode system in a chemical workstation with a brand of CHI760E, Chenhua. For the measurements, CTF samples $(2 \mathrm{~g})$ were firstly dispersed in $1 \%$ Nafion ethanol solution $(5 \mathrm{~mL})$ and then ultrasonic $20 \mathrm{~min}$. After that, $100 \mu \mathrm{L}$ suspension was deposited on ITO plate to act as the working electrode, while $\mathrm{Ag} / \mathrm{AgCl}$ electrode worked as the reference electrode and Pt flake acted as the counter electrode. $0.1 \mathrm{M} \mathrm{Na}_{2} \mathrm{SO}_{4}$ aqueous solution acted as electrolyte during the measurements. Electrochemical impedance spectroscopy (EIS) frequency ranged from $1 \mathrm{~Hz}$ to $10^{5} \mathrm{~Hz}$ and the photocurrents were measured under visible light irritation with interval of 30 second, while the Mott-Schottky curves were recorded at $1500 \mathrm{~Hz}, 2000 \mathrm{~Hz}$ and $2500 \mathrm{~Hz}$, respectively.

Photocatalytic experiment. Photocatalytic hydrogen evolution was conducted under visible light irradiation with the "sea water" containing $90 \mathrm{~mL}$ deionized water and $3.0 \mathrm{~g} \mathrm{NaCl}, 10 \mathrm{~mL}$ TEOA as sacrificial agent and 3\% Pt was supported as co-catalyst by chemical reduction of $\mathrm{NaBH}_{4}$. (CTF-HUST-3 sample in the previous report has used a different synthetic condition, and also the Pt nanoparticles were supported by using an in-situ deposition method for the photocatalytic hydrogen evolution experiment). The real sea water was from East China Sea at Xiamen, China. The apparent quantum yield (AQY) was obtained under the same conditions as the hydrogen evolution except the light at $420 \mathrm{~nm}$ or $500 \mathrm{~nm}$ as the irradiation source. The AQY was finally calculated according to the following equation:

$$
\begin{aligned}
\text { AQY }(\%) & =\frac{2 \times \text { number of evolved } \mathrm{H}_{2} \text { molecules }}{\text { number of incident photons }} \times 100 \% \\
& =\frac{2 \times \mathrm{CN}_{\mathrm{A}}}{\mathrm{Pt} \lambda / h c} \times 100 \%
\end{aligned}
$$

where $\mathrm{C}$ is the amount of $\mathrm{H}_{2} ; \lambda$ is the irradiation light wavelength (420 nm or $500 \mathrm{~nm}$ ), $\mathrm{N}_{\mathrm{A}}$ is the Avogadro constant; $h$ is the plank constant, $c$ is light speed, $\underline{t}$ is the light irradiation time, and $\mathrm{P}$ is the incident light intensity. 


\section{Synthesis of crystalline CTFs with heterogeneous nucleation agent}

\subsection{Synthesis of CTF-HUST-S1}

The mixture of terephthalaldehyde $(0.067 \mathrm{~g}, 0.5 \mathrm{mmol})$, terephthalamidine dihydrochloride (0.235 g, $1.0 \mathrm{mmol}), \mathrm{Cs}_{2} \mathrm{CO}_{3}(0.652 \mathrm{~g}, 2.0 \mathrm{mmol})$ and $\mathrm{NaCl}(5 \mathrm{~g})$ were dispersed in DMSO (20 $\mathrm{mL}$ ), and then the mixture was heated at $150{ }^{\circ} \mathrm{C}$ for $72 \mathrm{~h}$, and $160{ }^{\circ} \mathrm{C}$ for $24 \mathrm{~h}$. After cooling the reaction mixture to room temperature, the suspension was treated with filtration, washed with water twice, then with DMF once and finally washed with water thoroughly. After that, the powder sample was freeze-dried. The product CTF-HUST-S1 was obtained as a light yellow powder (0.178 g, yield: $85 \%)$. The synthesis process of the CTF-HUST-1 was the same with that of CTF-HUST-S1 in the absence of nucleation agent.

\subsection{Synthesis of CTF-HUST-S2}

The mixture of 4,4'-biphenyldicarboxaldehyde $(0.105 \mathrm{~g}, 0.5 \mathrm{mmol})$, terephthalamidine dihydrochloride $(0.235 \mathrm{~g}, 1.0 \mathrm{mmol}), \mathrm{Cs}_{2} \mathrm{CO}_{3}(0.652 \mathrm{~g}, 2.0 \mathrm{mmol})$ and $\mathrm{NaCl}(5 \mathrm{~g})$ were dispersed in DMSO $(20 \mathrm{~mL})$, and then the mixture was heated at $150{ }^{\circ} \mathrm{C}$ for $72 \mathrm{~h}$, and $160{ }^{\circ} \mathrm{C}$ for $24 \mathrm{~h}$. After cooling the reaction mixture, the suspension was treated with filtration, washed with water twice, then with DMF once and finally washed with water thoroughly. After that, the powder was freeze-dried. The product CTF-HUST-S2 was obtained as light yellow powder (0.192 g, yield: $77 \%)$.

Control experiments were conducted using different nucleation agent. With the same synthesis process, the salt was varied by $\mathrm{KCl}, \mathrm{LiCl}, \mathrm{Na}_{2} \mathrm{SO}_{4}$ and $\mathrm{NaBr}$ with the same amount and the corresponding CTFs were named as CTF-HUST-S2-KCl, CTF-HUST-S2-LiCl, CTF-HUSTS2- $\mathrm{Na}_{2} \mathrm{SO}_{4}$ and CTF-HUST-S2-NaBr, respectively. The synthesis process of the CTF-HUST2 was the same with that of CTF-HUST-S2 in the absence of nucleation agent.

\subsection{Synthesis of CTF-HUST-S3}

The mixture of tris(4-formylphenyl)amine $(0.099 \mathrm{~g}, \quad 0.3 \mathrm{mmol})$, terephthalamidine dihydrochloride $(0.212 \mathrm{~g}, 0.9 \mathrm{mmol}), \mathrm{Cs}_{2} \mathrm{CO}_{3}(0.586 \mathrm{~g}, 1.8 \mathrm{mmol})$ and $\mathrm{NaCl}(10 \mathrm{~g})$ were dispersed in DMSO $(20 \mathrm{~mL})$, and then the mixture was heated at $150{ }^{\circ} \mathrm{C}$ for $72 \mathrm{~h}$, and $160^{\circ} \mathrm{C}$ for $24 \mathrm{~h}$. After cooling the reaction mixture, the suspension was treated with filtration, washed 
with water twice, then with DMF once and finally washed with water thoroughly. After that, the powder was freeze-dried. The product CTF-HUST-S3 was obtained as a light powder (0.185 g, yield: $81 \%)$. The synthesis process of the CTF-HUST-3 was the same with that of CTF-HUST-S3 in the absence of nucleation agent.

\subsection{Synthesis of CTF-HUST-S4}

The mixture of [2,2'-bipyridine]-5,5'-dicarboxaldehyde $(0.0796 \mathrm{~g}, 0.375 \mathrm{mmol})$, terephthalamidine dihydrochloride $(0.176 \mathrm{~g}, 0.75 \mathrm{mmol}), \mathrm{Cs}_{2} \mathrm{CO}_{3}(0.489 \mathrm{~g}, 1.5 \mathrm{mmol})$ and sodium chloride $(7.5 \mathrm{~g})$ were dispersed in DMSO $(15 \mathrm{~mL})$, and then the mixture was heated at $150{ }^{\circ} \mathrm{C}$ for $72 \mathrm{~h}$, and $160{ }^{\circ} \mathrm{C}$ for $24 \mathrm{~h}$. After cooling the reaction mixture, the suspension was treated with filtration, washed with water twice, then with DMF once and finally washed with water thoroughly. After that, the powder was freeze-dried. The product CTF-HUST-S4 was obtained as a yellow powder ( $0.156 \mathrm{~g}$, yield: $84 \%)$. The synthesis process of the CTF-HUST-4 was the same with that of CTF-HUST-S4 in the absence of nucleation agent.

\subsection{Synthesis of CTF-HUST-S5}

The mixture of thieno[3,2-b]thiophene-2,5-dicarboxaldehyde $(0.098 \mathrm{~g}, 0.5 \mathrm{mmol})$, terephthalamidine dihydrochloride $(0.235 \mathrm{~g}, 1.0 \mathrm{mmol}), \mathrm{Cs}_{2} \mathrm{CO}_{3}(0.652 \mathrm{~g}, 2.0 \mathrm{mmol})$ and $\mathrm{NaCl}$ (5 g) were dispersed in DMSO $(20 \mathrm{~mL})$, and then the mixture was heated at $150{ }^{\circ} \mathrm{C}$ for $72 \mathrm{~h}$, $160{ }^{\circ} \mathrm{C}$ for $24 \mathrm{~h}$. After cooling the reaction, the suspension was treated with filtration, washed with water twice, then with DMF once and finally washed with water thoroughly. After that, the powder was freeze-dried. The product CTF-HUST-S5 was obtained as a brown red powder (0.185 g, yield: $77 \%)$. The synthesis process of the CTF-HUST-5 was the same with that of CTF-HUST-S5 in the absence of nucleation agent.

\subsection{Synthesis of CTF-HUST-S6}

The mixture of 4-[3,5-bis(4-formylphenyl)phenyl]benzaldehyde $(0.117 \mathrm{~g}, 0.3 \mathrm{mmol})$, terephthalamidine dihydrochloride $(0.212 \mathrm{~g}, 0.9 \mathrm{mmol}), \mathrm{Cs}_{2} \mathrm{CO}_{3}(0.586 \mathrm{~g}, 1.8 \mathrm{mmol})$ and $\mathrm{NaCl}$ $(10 \mathrm{~g})$ were dispersed in DMSO $(20 \mathrm{~mL})$, and then the mixture was heated at $150{ }^{\circ} \mathrm{C}$ for $72 \mathrm{~h}$, and $160{ }^{\circ} \mathrm{C}$ for $24 \mathrm{~h}$. After cooling the reaction, the suspension was treated with filtration, 
washed with water twice, then with DMF once and finally washed with water thoroughly. After that, the powder was freeze-dried. The product CTF-HUST-S6 was obtained as a light powder (0.199 g, yield: $81 \%)$. The synthesis process of the CTF-HUST-6 was the same with that of CTF-HUST-S6 in the absence of nucleation agent. 


\section{Evidences for the proposed mechanism}
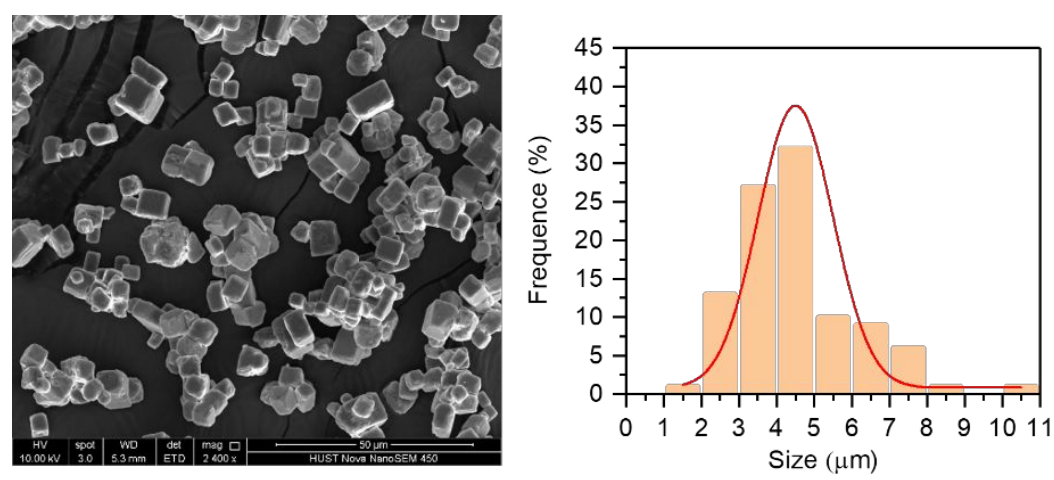

Figure S1 SEM images and the size distribution of $\mathrm{NaCl}$.
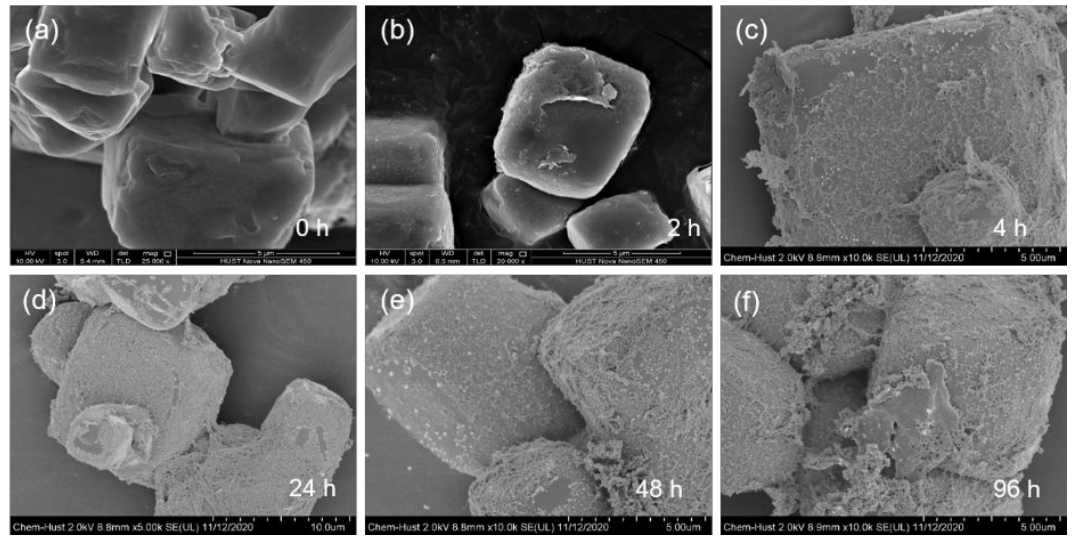

Figure S2 The SEM images of $\mathrm{NaCl}$ surface disclosed at different reaction time.
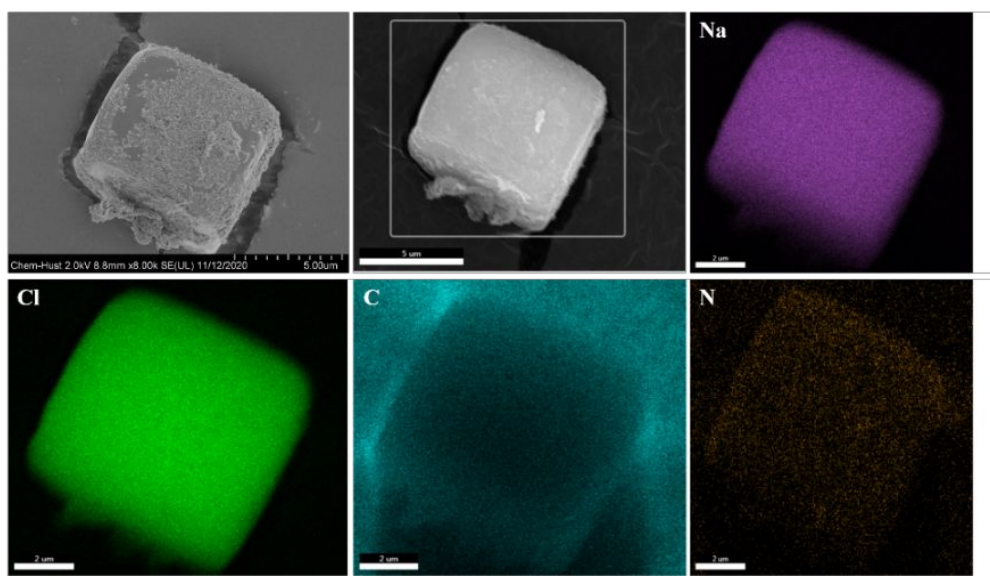

Figure S3 Elemental mapping of $\mathrm{NaCl}$ surface at the reaction $96 \mathrm{~h}$ without any posttreatment.

At the beginning of the polycondensation, the lattice of salt can induce the nucleation of the CTFs and the large surface of salt serve as the reaction place. With the reaction time prolonged, the crystalline salt could induce the further growth of the CTF layers along the 
lattice planes. Hence, the salt acts the heterogeneous nucleation agent.
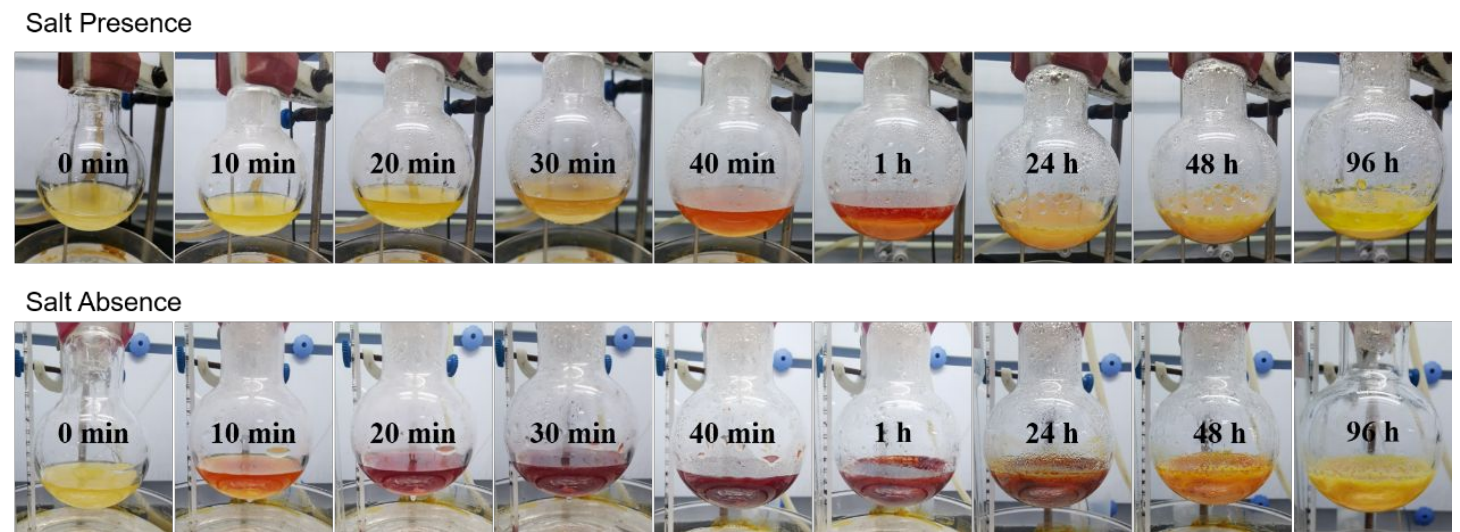

Figure S4. Optical photos of the reaction system at different reaction time. 
4. PXRD patterns of CTF-HUST-S2 synthesized in different nucleation agents.

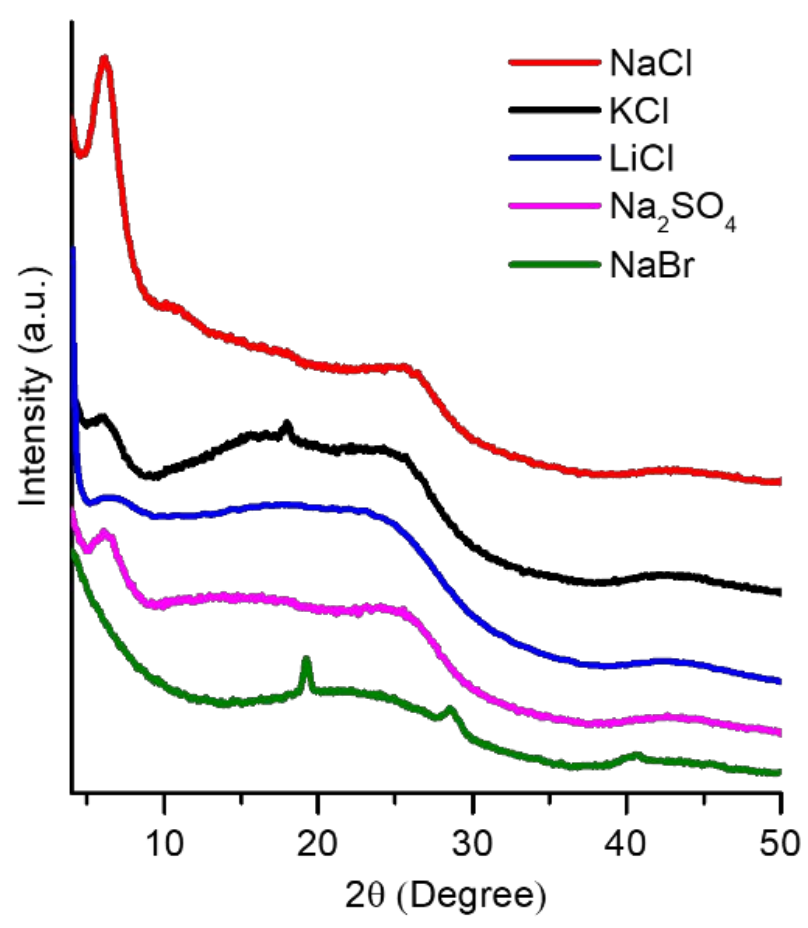

Figure S5. PXRD patterns of the CTF-HUST-S2 synthesized in different nucleation agent.

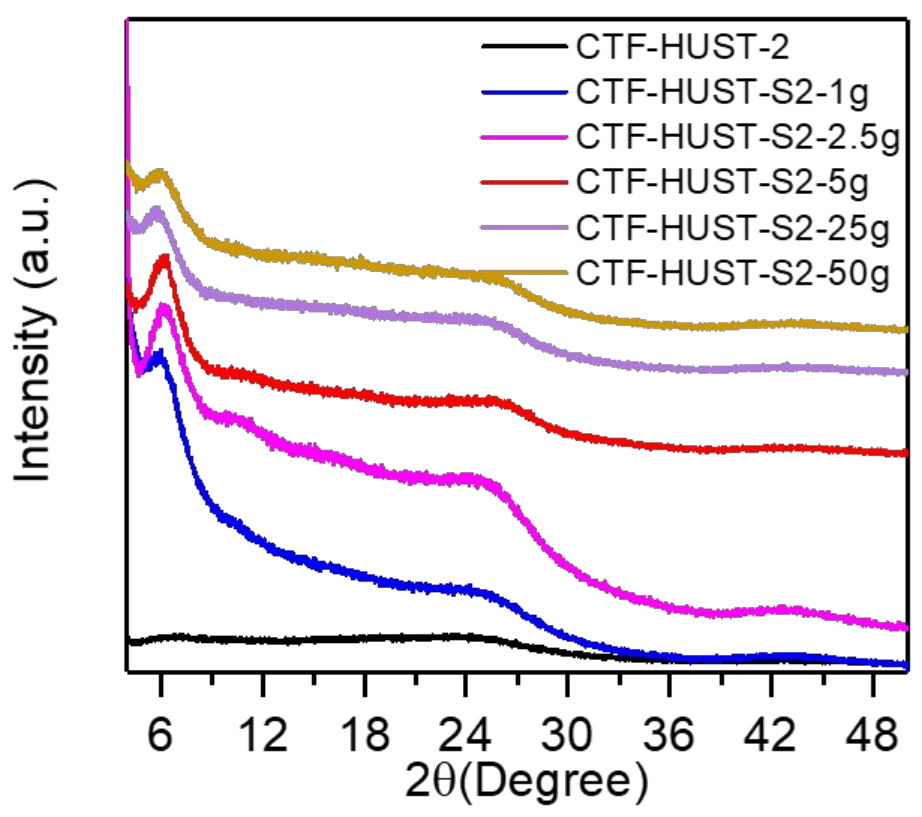

Figure S6. PXRD patterns of the CTF-HUST-S2 synthesized with different amount $\mathrm{NaCl}$. 


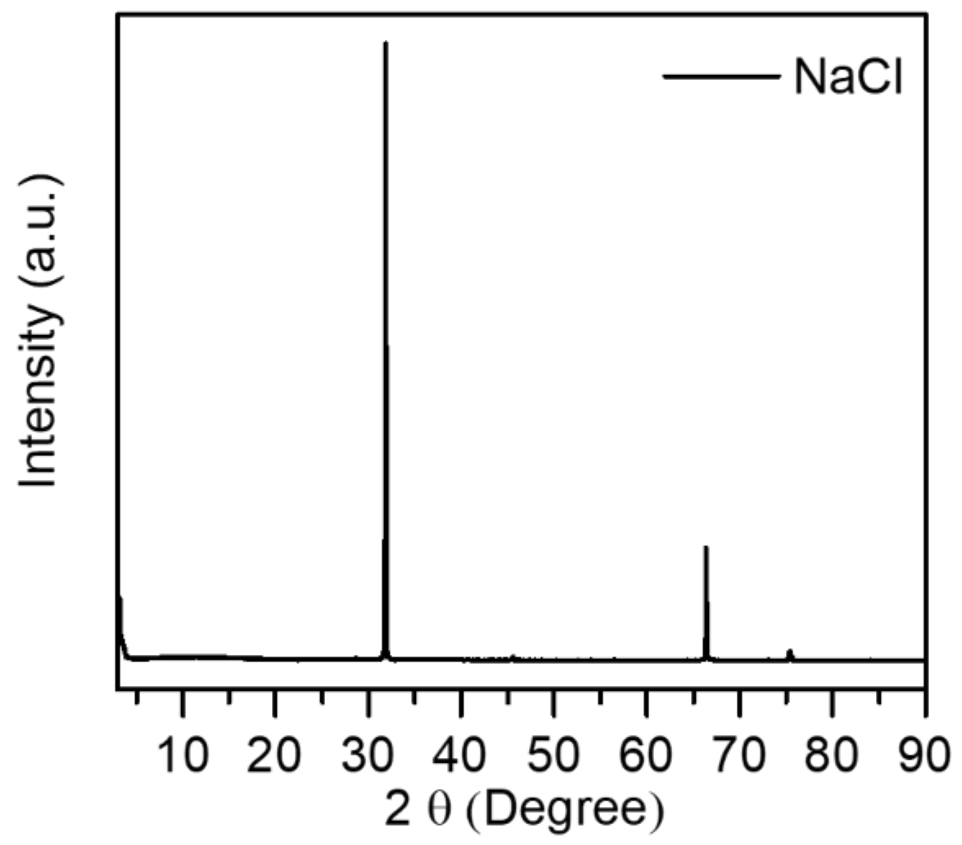

Figure S7. PXRD pattern of $\mathrm{NaCl}$. 
5. PXRD simulation of the crystalline structures

(a)

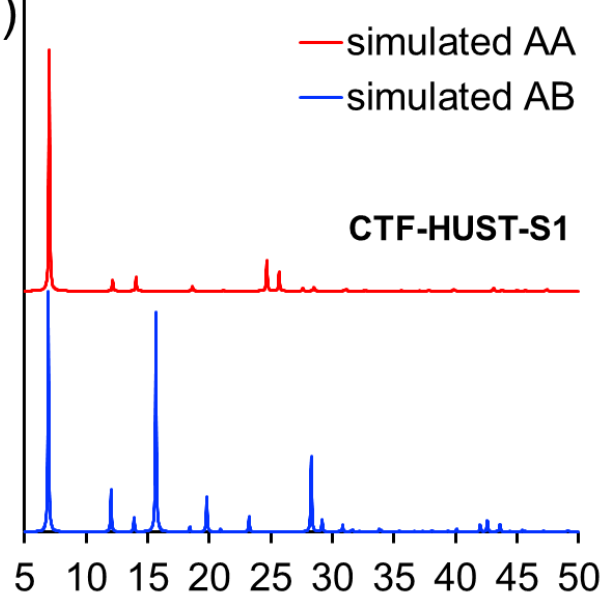

(c)

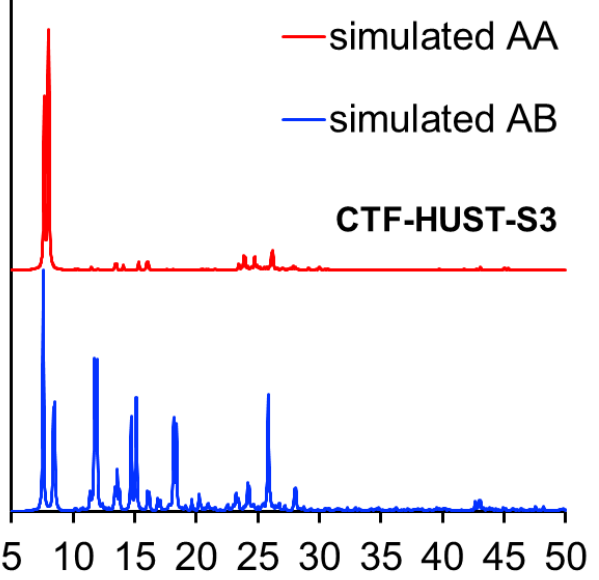

(e)

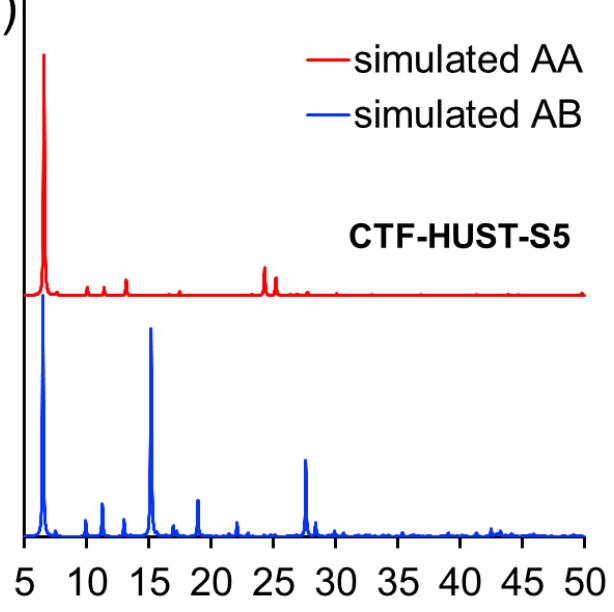

(b)

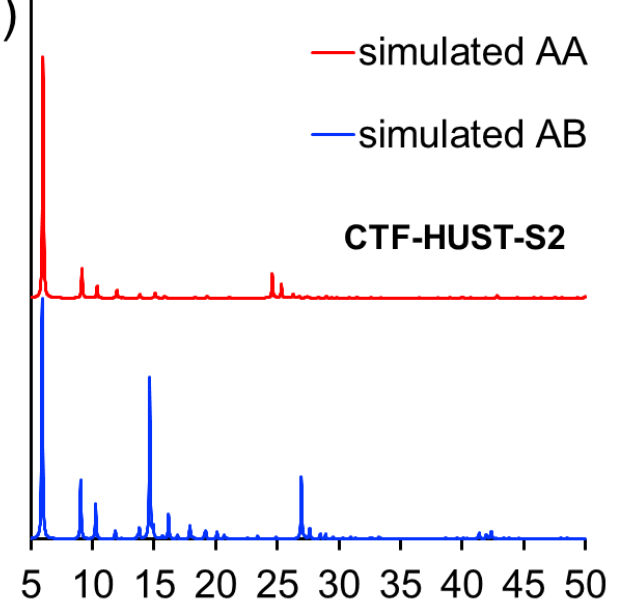

(d)

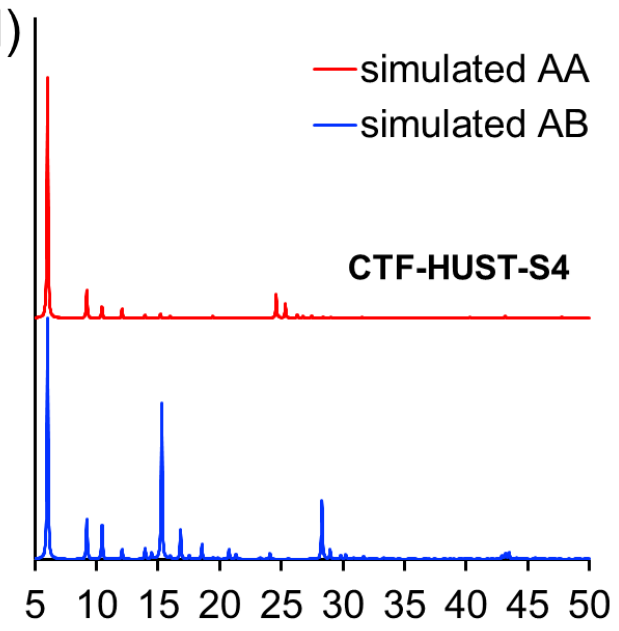

(f)

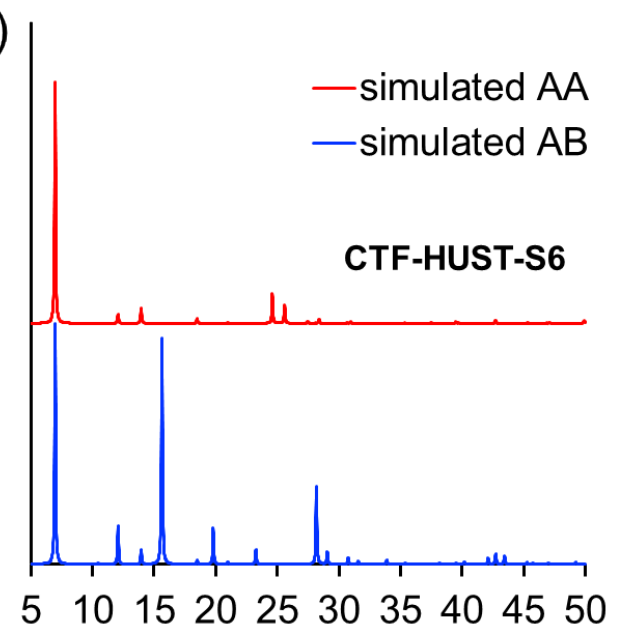

Figure S8. Simulated PXRD of AA and AB stacking patterns for CTF-HUST-S1 to CTF-

HUST-S6. 


\section{HR-TEM images of CTF-HUST-Sx}
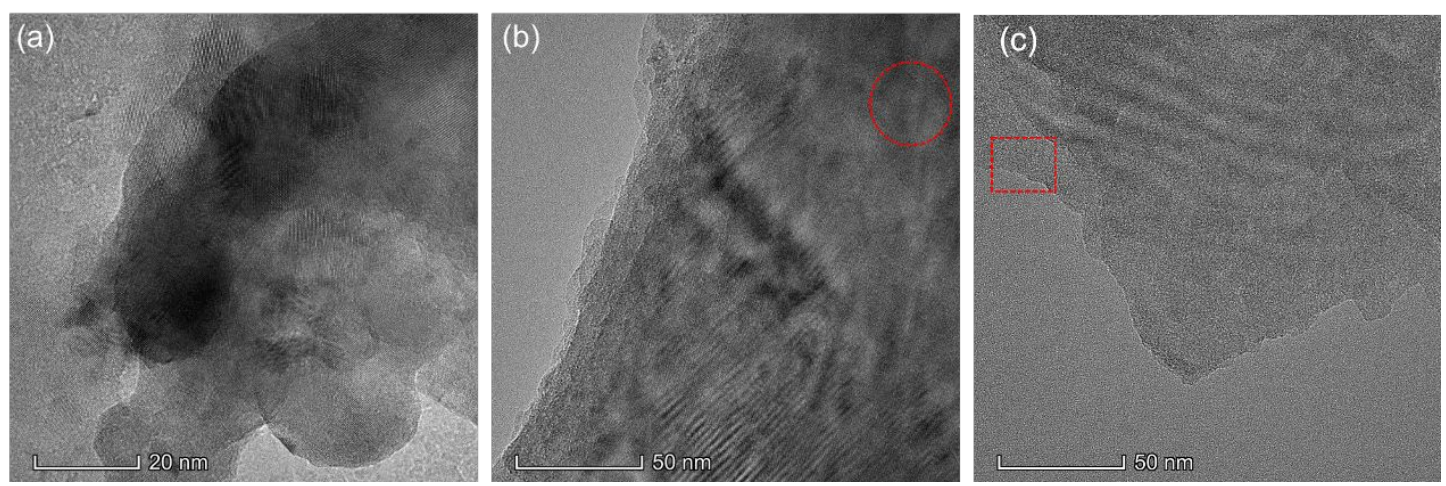

Figure S9. HR-TEM images of the crystalline CTF-HUST-S1 observed at other regions.
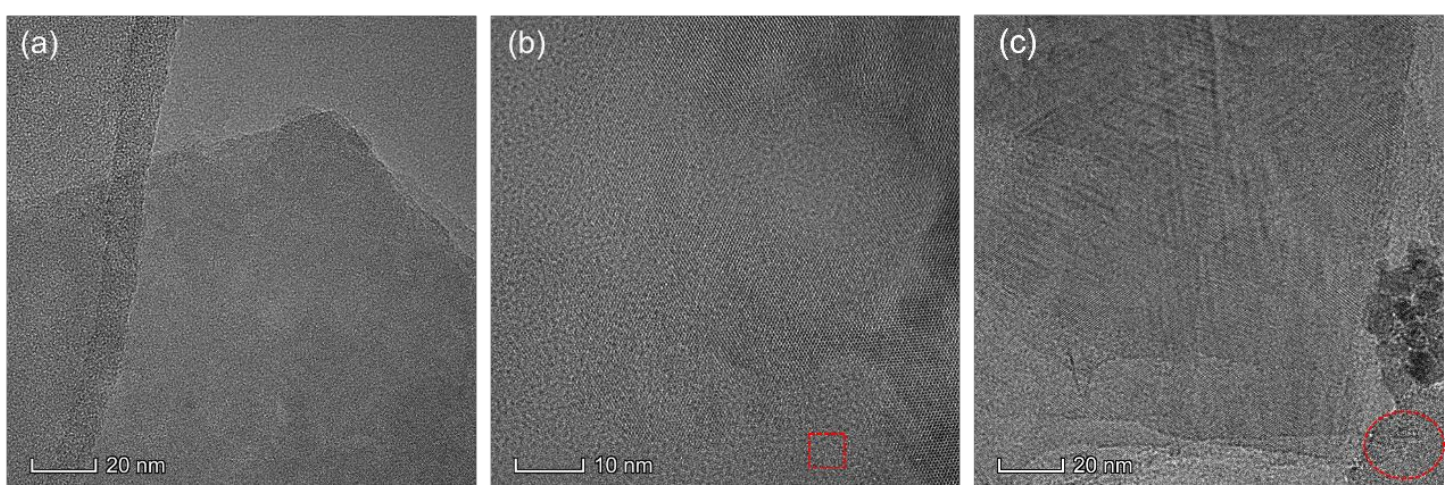

Figure S10. HR-TEM images of the crystalline CTF-HUST-S2 observed at other regions.
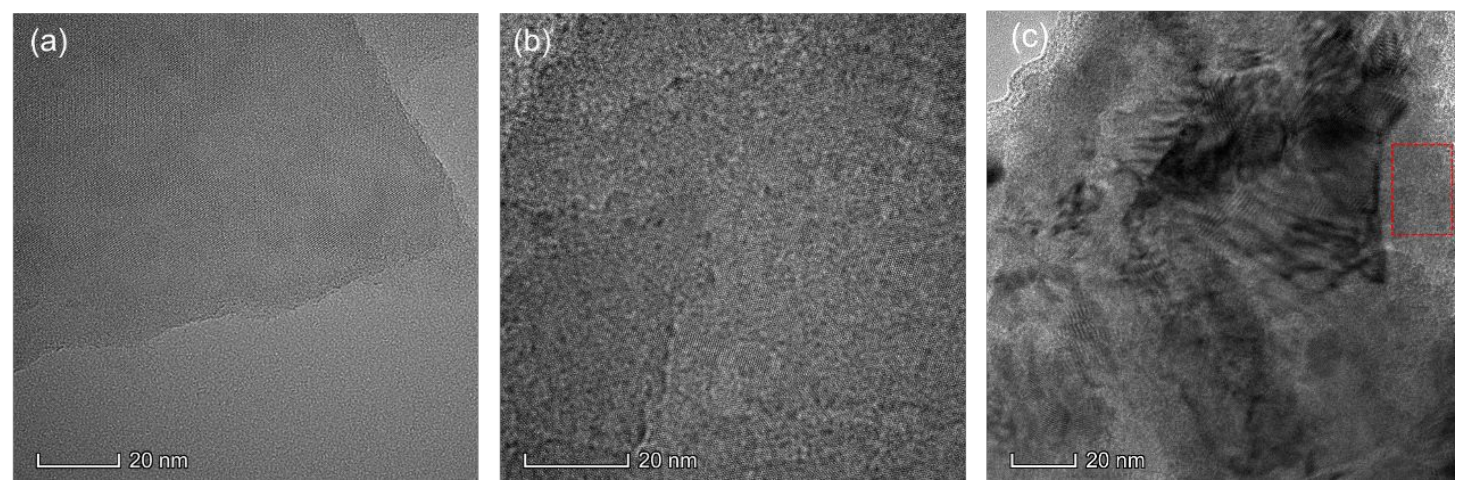

Figure S11. HR-TEM images of the crystalline CTF-HUST-S3 observed at other regions.
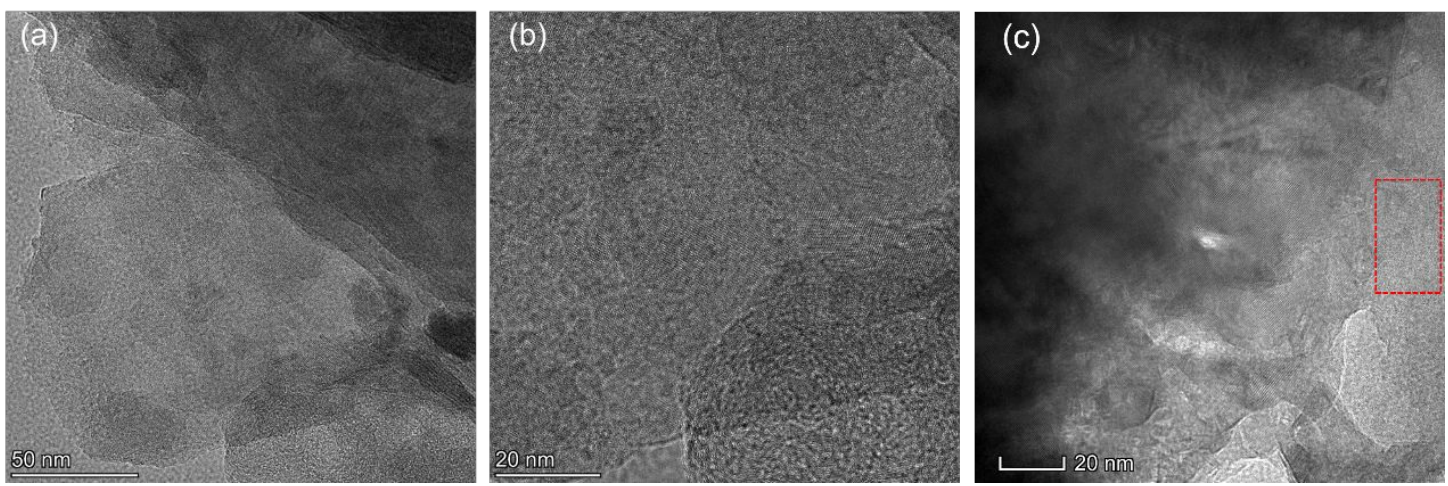

Figure S12. HR-TEM images of the crystalline CTF-HUST-S4 observed at other regions. 

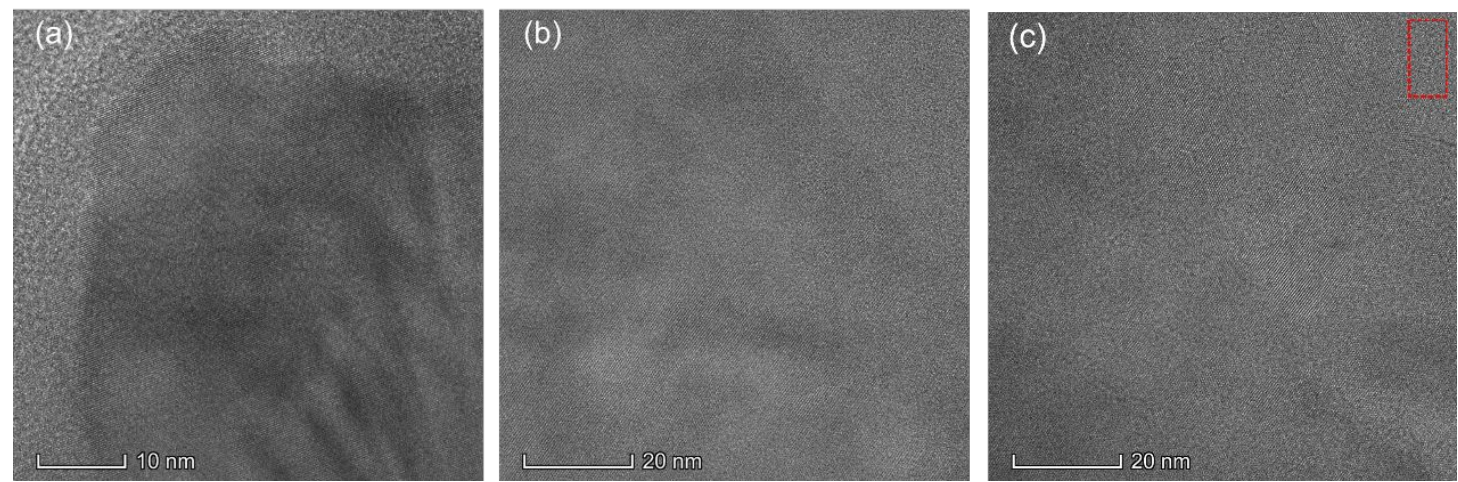

Figure S13. HR-TEM images of the crystalline CTF-HUST-S5 observed at other regions.
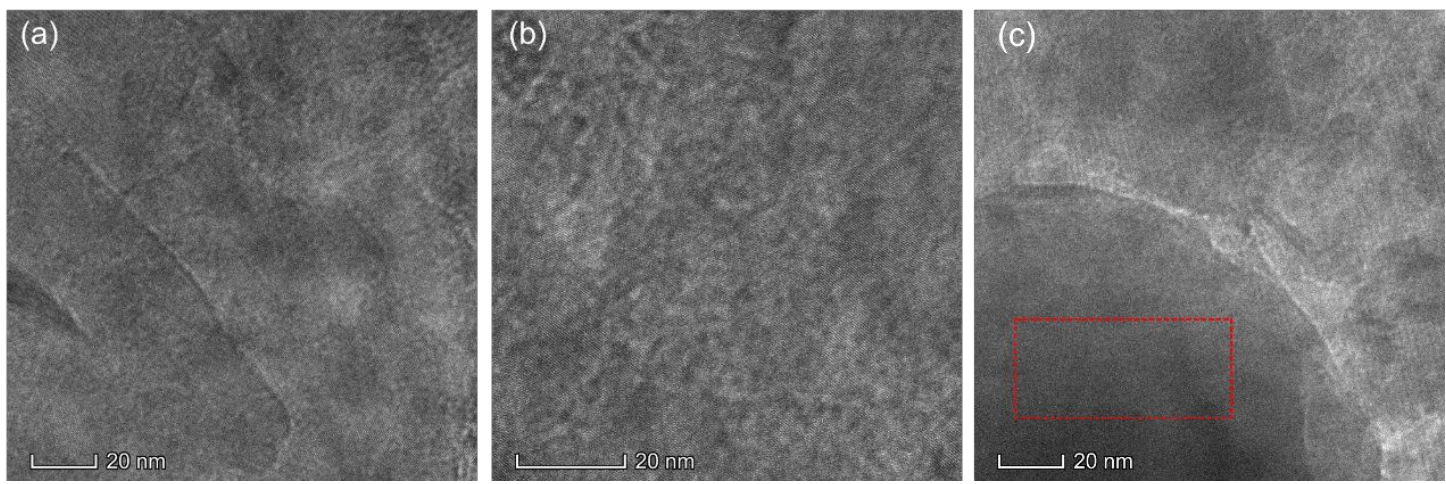

Figure S14. HR-TEM images of the crystalline CTF-HUST-S6 observed at other regions.

The amorphous domains are circled in Figure S9-S14.
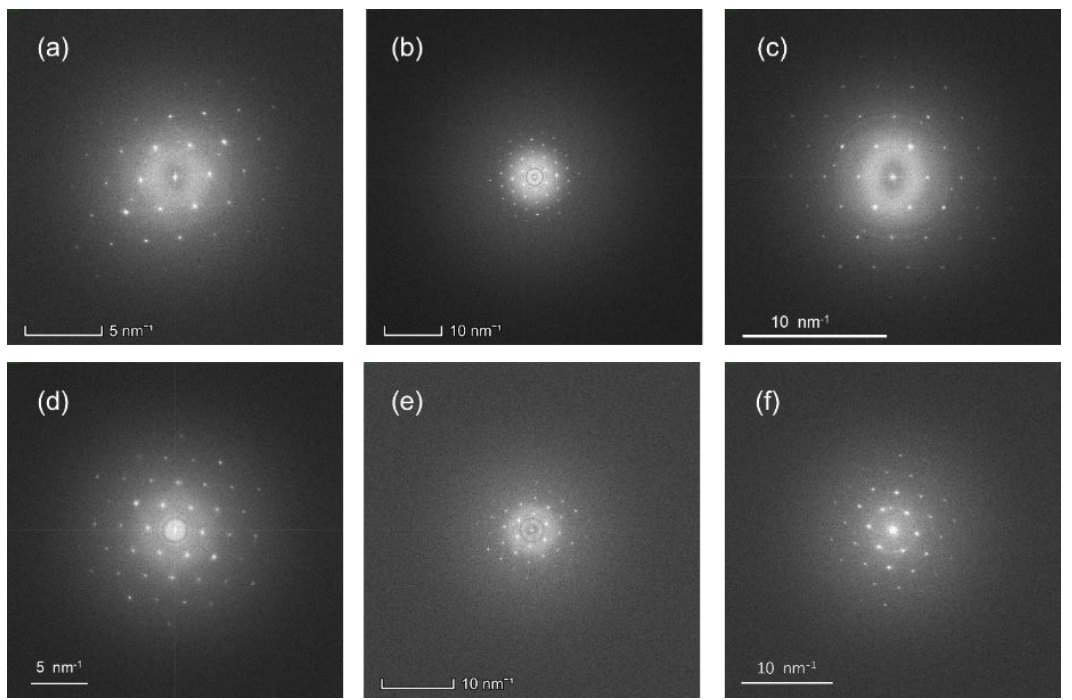

Figure S15. FFT images of CTF-HUST-Sx: (a) CTF-HUST-S1; (b) CTF-HUST-S2; (c) CTFHUST-S3; (d) CTF-HUST-S4; (e) CTF-HUST-S5; (f) CTF-HUST-S6 
7. FT-IR spectra of CTF-HUST-Sx

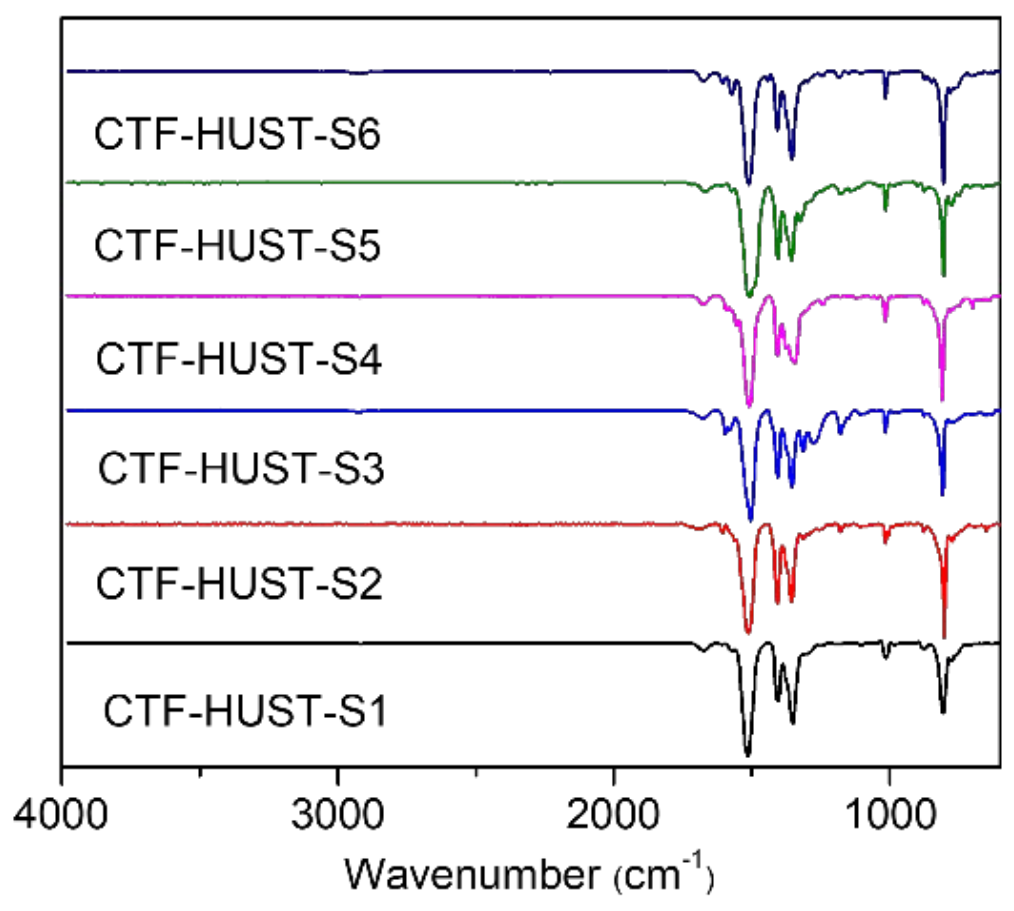

Figure S16. FT-IR spectra of CTF-HUST-Sx. 


\section{XPS of CTF-HUST-Sx}

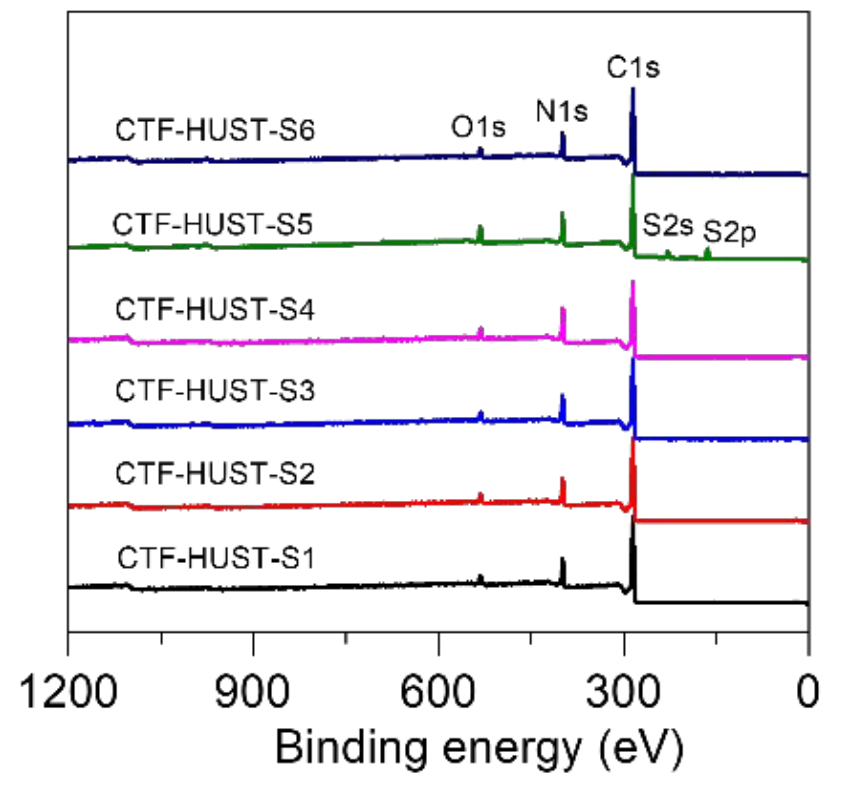

Figure S17. XPS survey of CTF-HUST-Sx

(a)

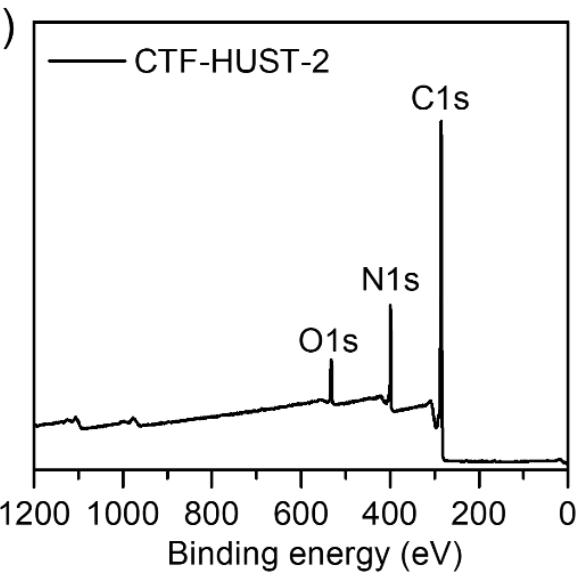

(b)

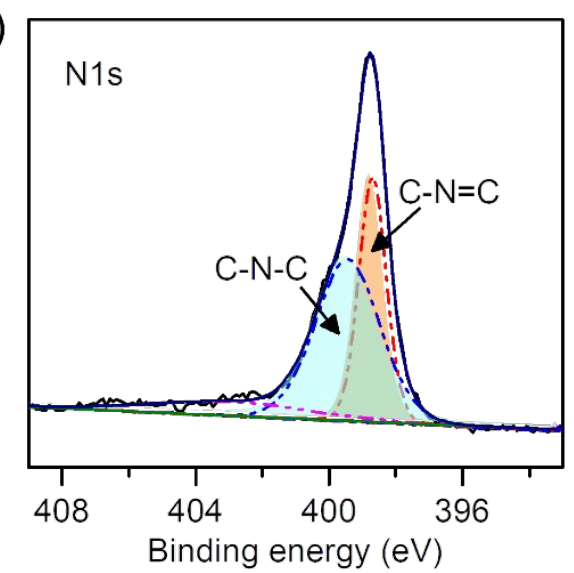

Figure S18. XPS survey (a) and high resolution XPS of N1s (b) of CTF-HUST-2 


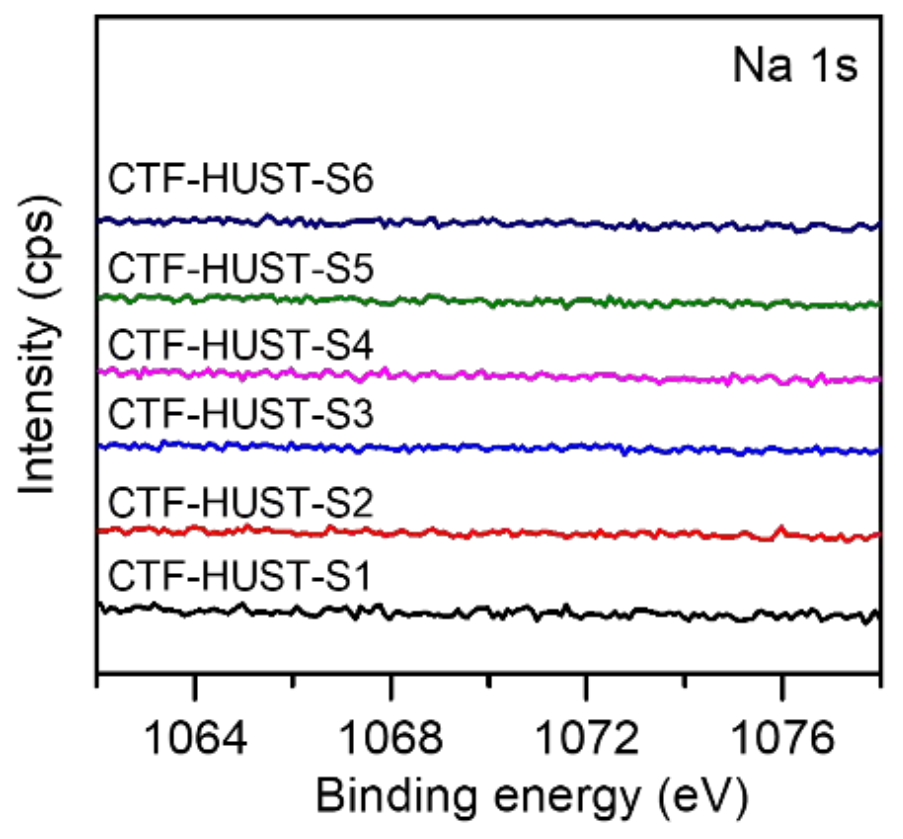

Figure S19. High resolution XPS of Na1s of CTF-HUST-Sx 


\section{Thermal stability of CTF-HUST-Sx}

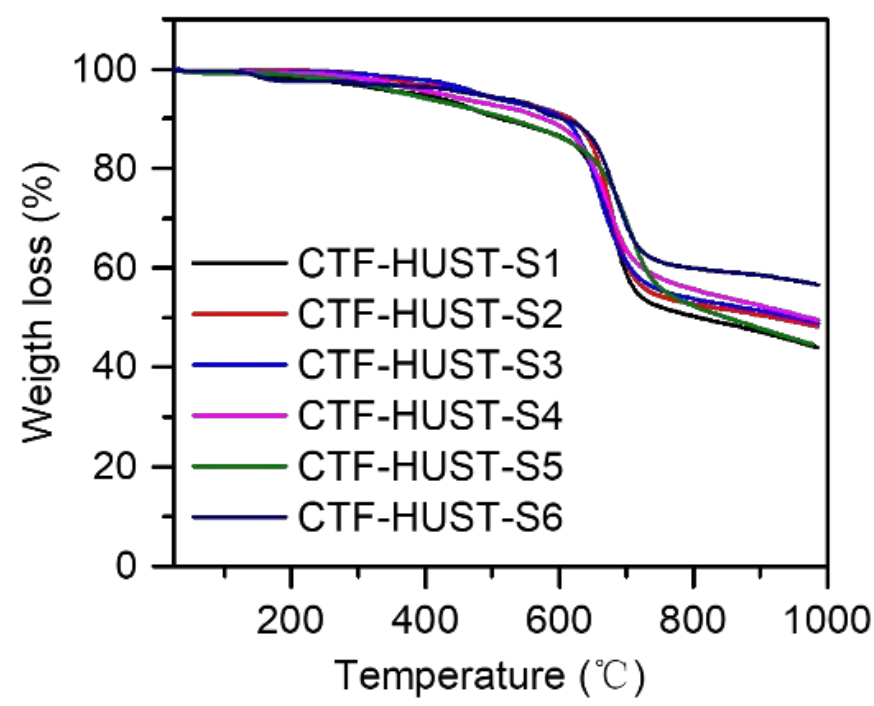

Figure S20. TGA curves of CTF-HUST-Sx under nitrogen atmosphere.
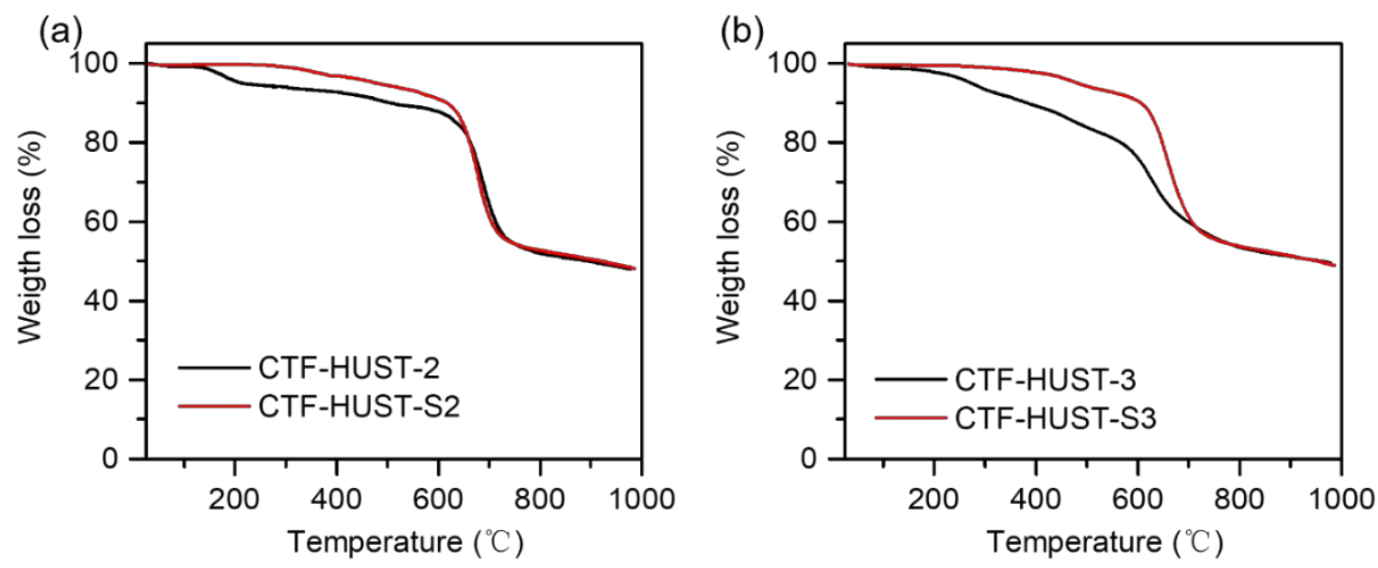

Figure S21. TGA curves of CTF-HUST-S2 and CTF-HUST-2 (a) and CTF-HUST-S3 and CTF-

HUST-3 (b) under nitrogen atmosphere. 
10. Morphology of CTF-HUST-Sx
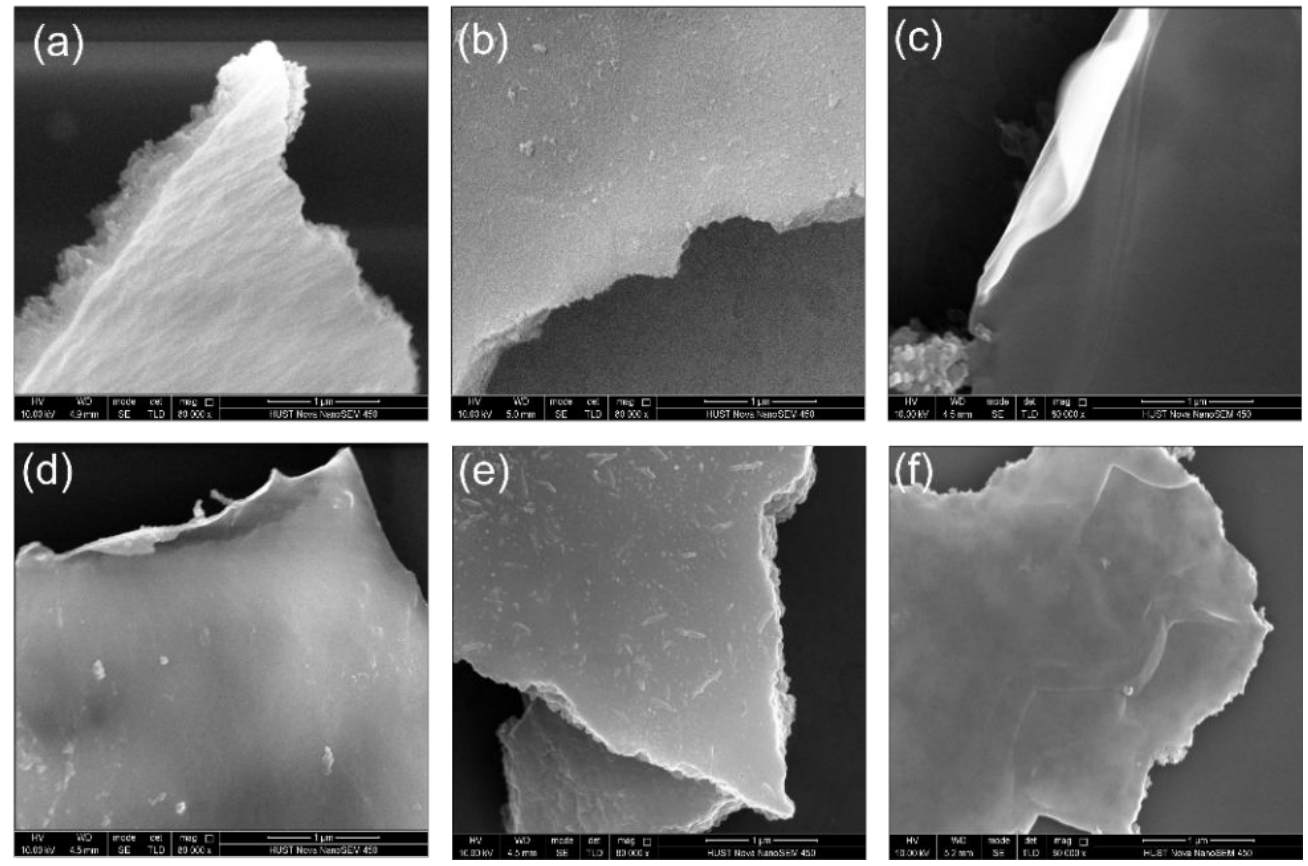

Figure S22. SEM images of CTFs: (a) CTF-HUST-S1, (b) CTF-HUST-S2, (c) CTF-HUST-S3, (d) CTF-HUST-S4, (e) CTF-HUST-S5, (f) CTF-HUST-S6.
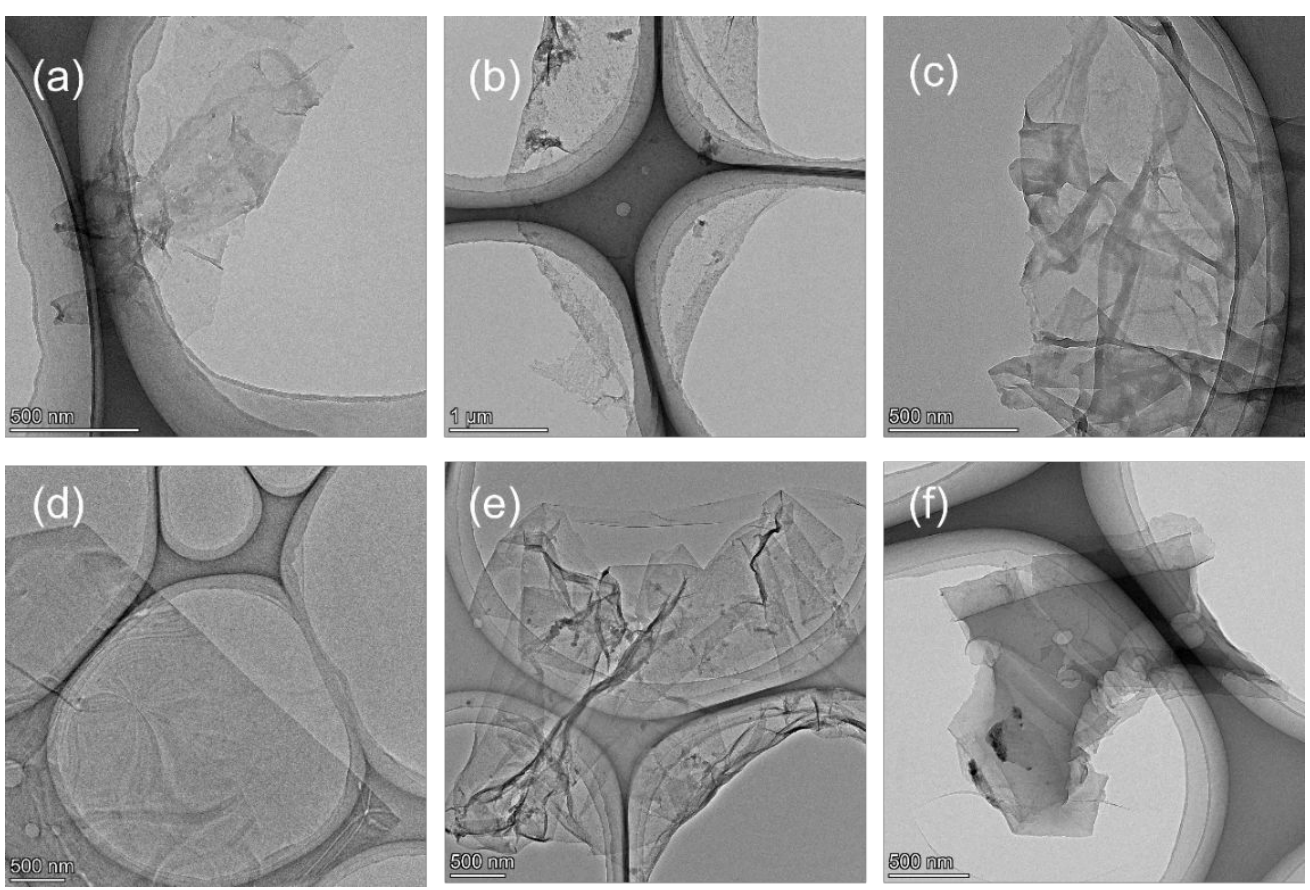

Figure S23. TEM images of the crystalline CTFs: (a) CTF-HUST-S1, (b) CTF-HUST-S2, (c) CTF-HUST-S3, (d) CTF-HUST-S4, (e) CTF-HUST-S5, (f) CTF-HUST-S6 
(a)

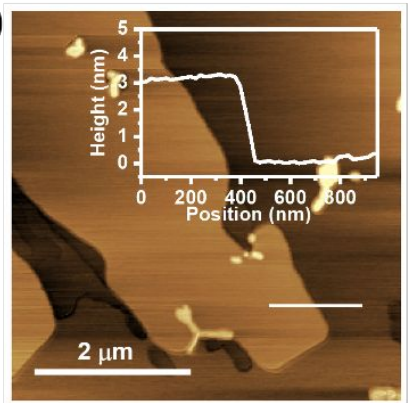

(d)

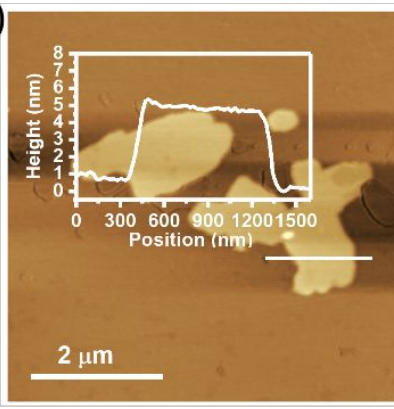

(b)

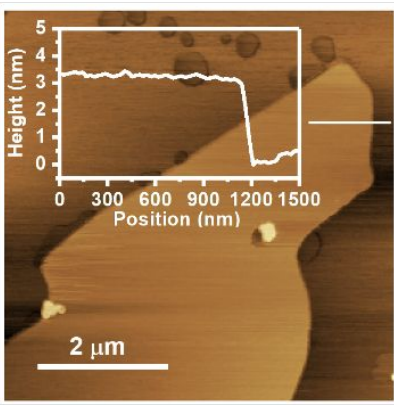

(e)

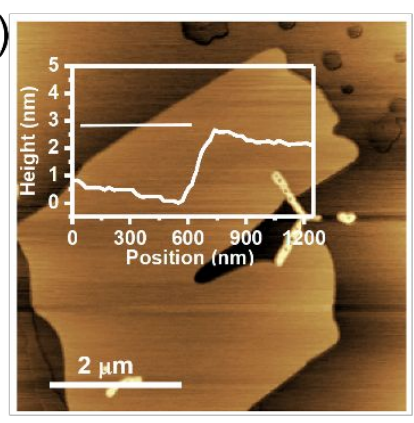

(c)

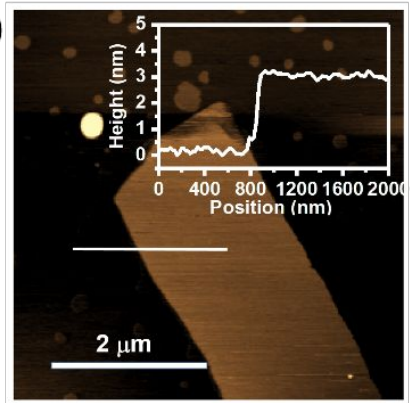

(f)

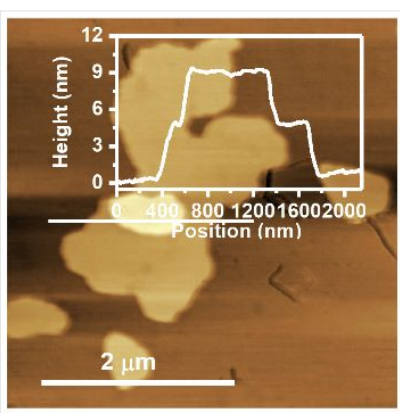

Figure S24. AFM images of the crystalline CTFs: (a) CTF-HUST-S1, (b) CTF-HUST-S2, (c) CTF-HUST-S3, (d) CTF-HUST-S4, (e) CTF-HUST-S5, (f) CTF-HUST-S6 
11. Dispersability of CTF-HUST-Sx

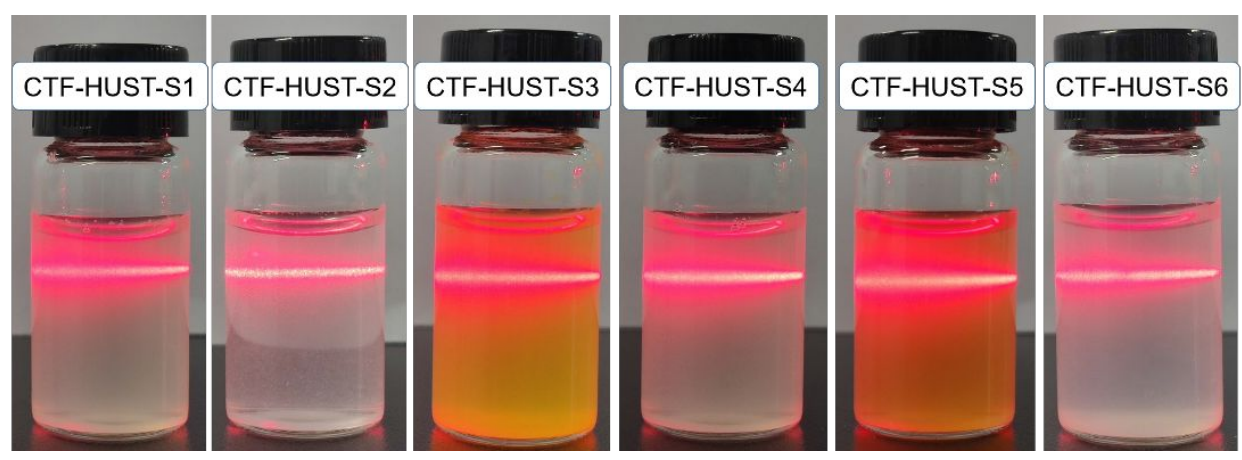

Figure S25. Digital photograph of the dispersion of CTF-HUST-Sx in water with the concertation of $0.1 \mathrm{mg} / \mathrm{mL}$.

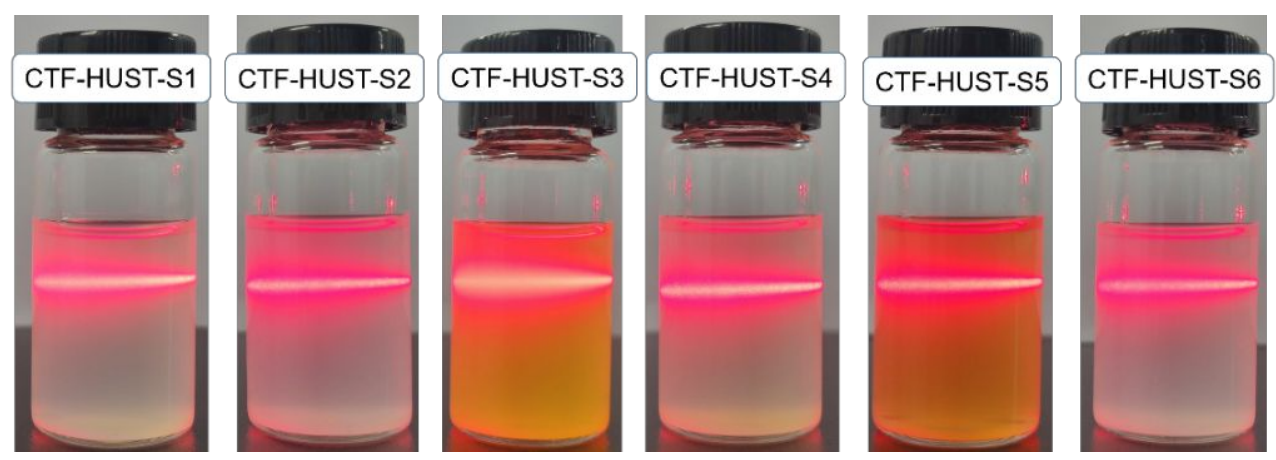

Figure S26. Digital photograph of dispersion of CTF-HUST-Sx in ethanol with the concertation of $0.1 \mathrm{mg} / \mathrm{mL}$. 
12. UV-visible absorption and optical band gaps of CTF-HUST-Sx
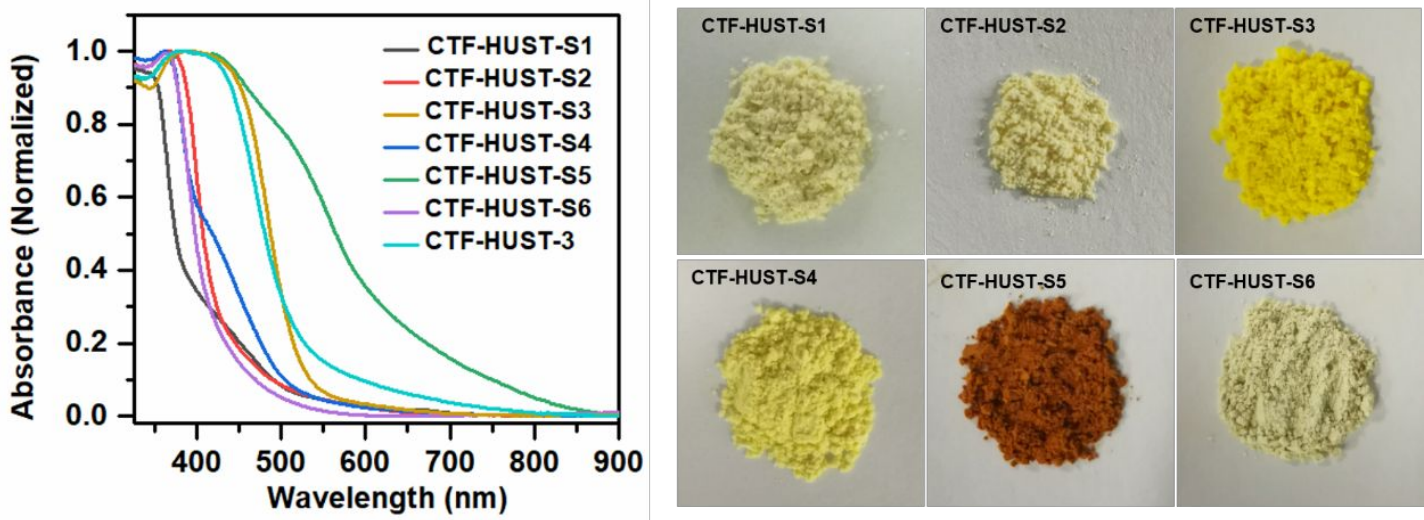

Figure S27. UV-visible light absorption spectra and the digital photograph of CTFs.
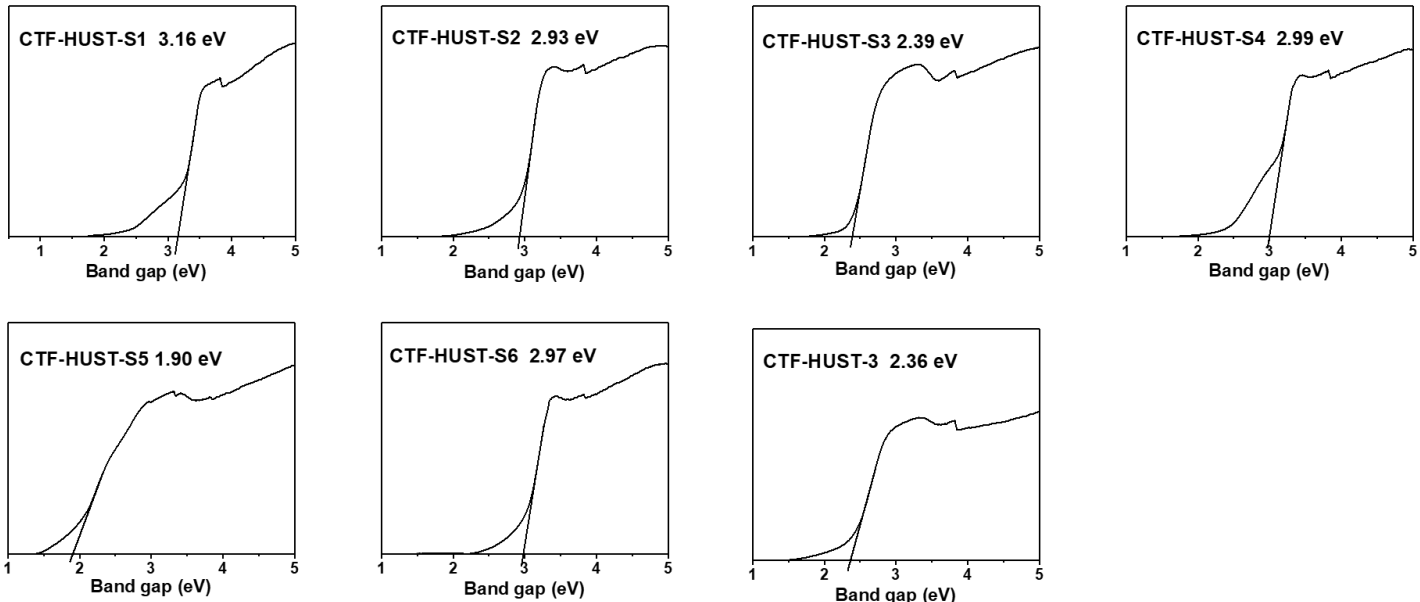

Figure S28. Band gap of CTF-HUST-Sx calculated by Kubelka-Munk function based on DRS. 
13. Energy level alignments of CTF-HUST-Sx
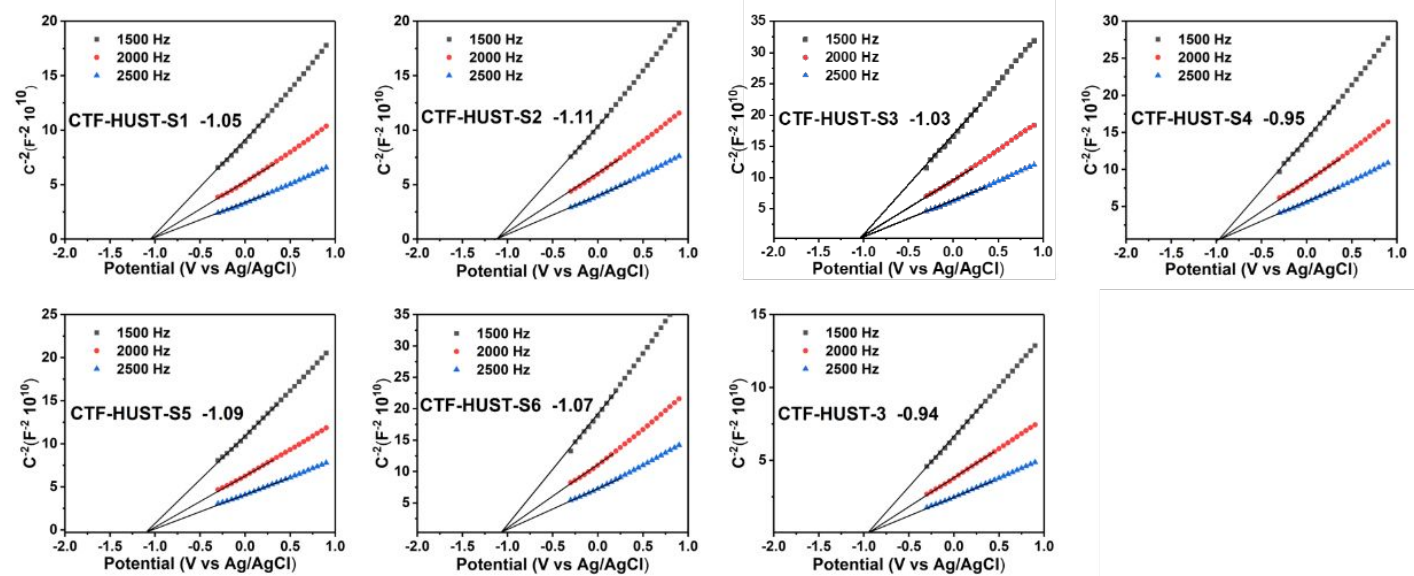

Figure S29. Mott-Schottky test results of CTF-HUST-Sx.

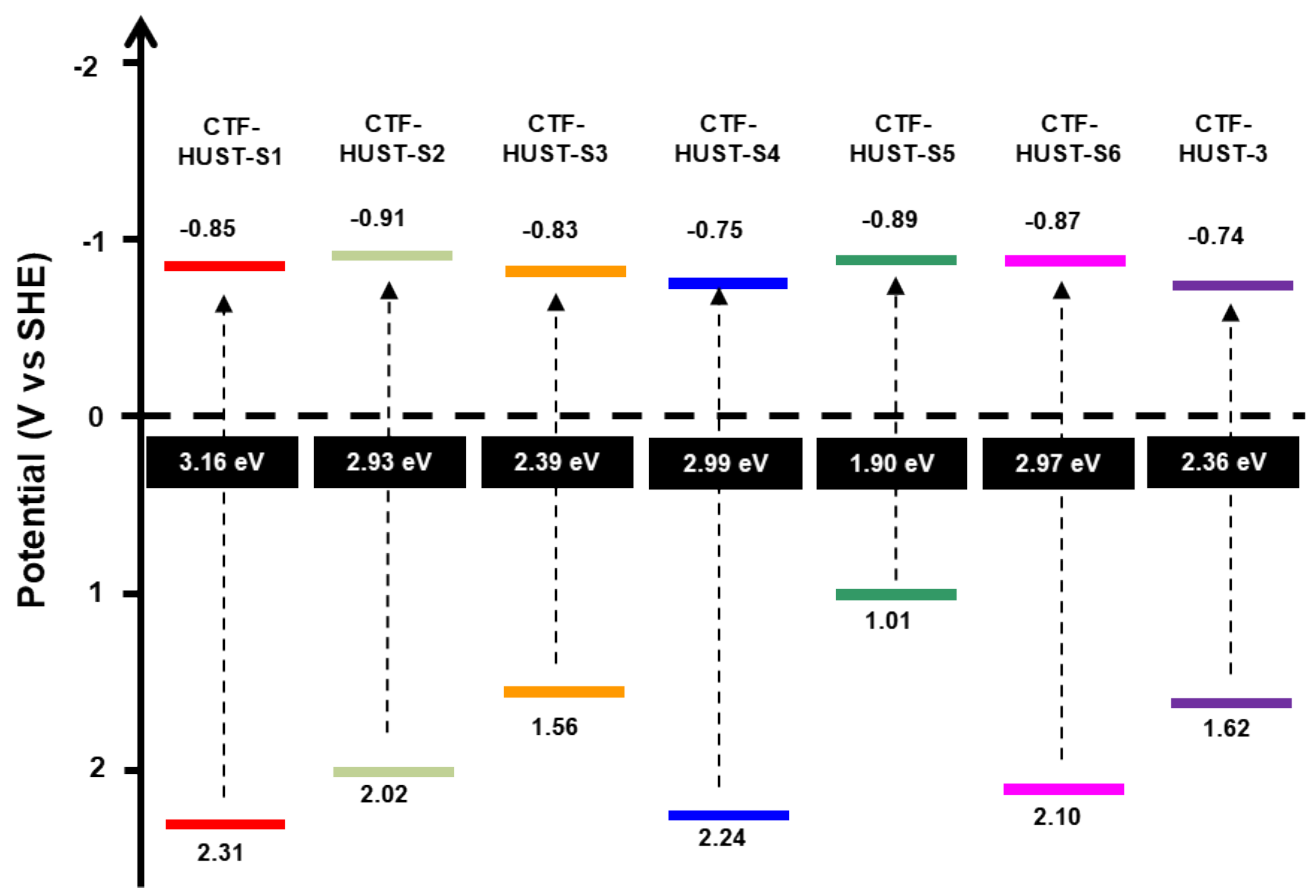

Figure S30. Energy level alignments of CTFs calculated from experimental results 
14. Photoluminescence and electrochemical performance of CTF-HUST-Sx

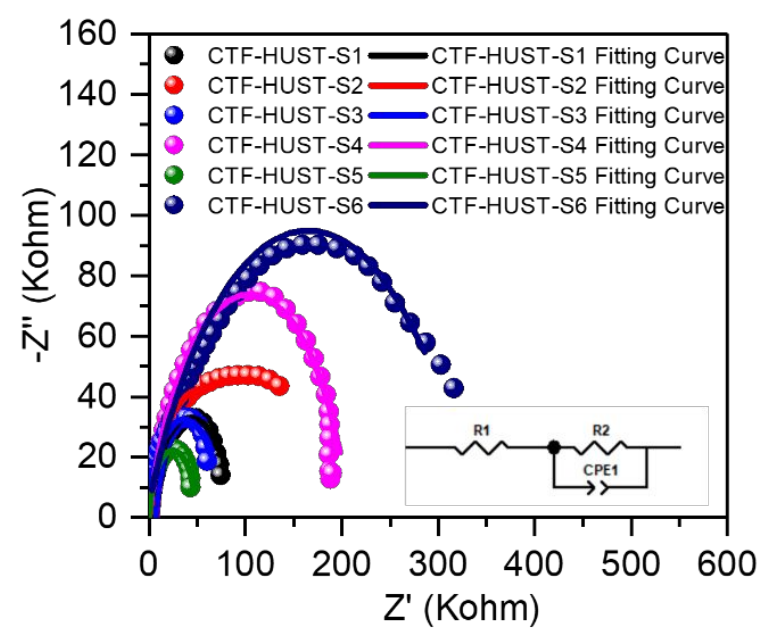

Figure S31. EIS Nyquist plots of CTF-HUST-Sx

Table S1 Parameters of equivalent circuit for the impedance data of CTF-HUST-Sx and CTF-

HUST-3

\begin{tabular}{ccccc}
\hline Sample & R1 (ohm) & R2 (ohm) & CPE-T $(\mathrm{F})$ & CPE-P \\
\hline CTF-HUST-S1 & 27.83 & 84616 & $4.501 \mathrm{E}^{-5}$ & 0.846 \\
CTF-HUST-S2 & 28.94 & 188820 & $3.091 \mathrm{E}^{-5}$ & 0.717 \\
CTF-HUST-S3 & 26.35 & 60548 & $1.598 \mathrm{E}^{-5}$ & 0.929 \\
CTF-HUST-S4 & 33.91 & 185650 & $3.571 \mathrm{E}^{-6}$ & 0.84912 \\
CTF-HUST-S5 & 31.99 & 46371 & $1.061 \mathrm{E}^{-5}$ & 0.93762 \\
CTF-HUST-S6 & 28.61 & 244920 & $2.712 \mathrm{E}^{-6}$ & 0.83488 \\
CTF-HUST-3 & 27.66 & 89499 & $3.439 \mathrm{E}^{-6}$ & 0.90329 \\
\hline
\end{tabular}




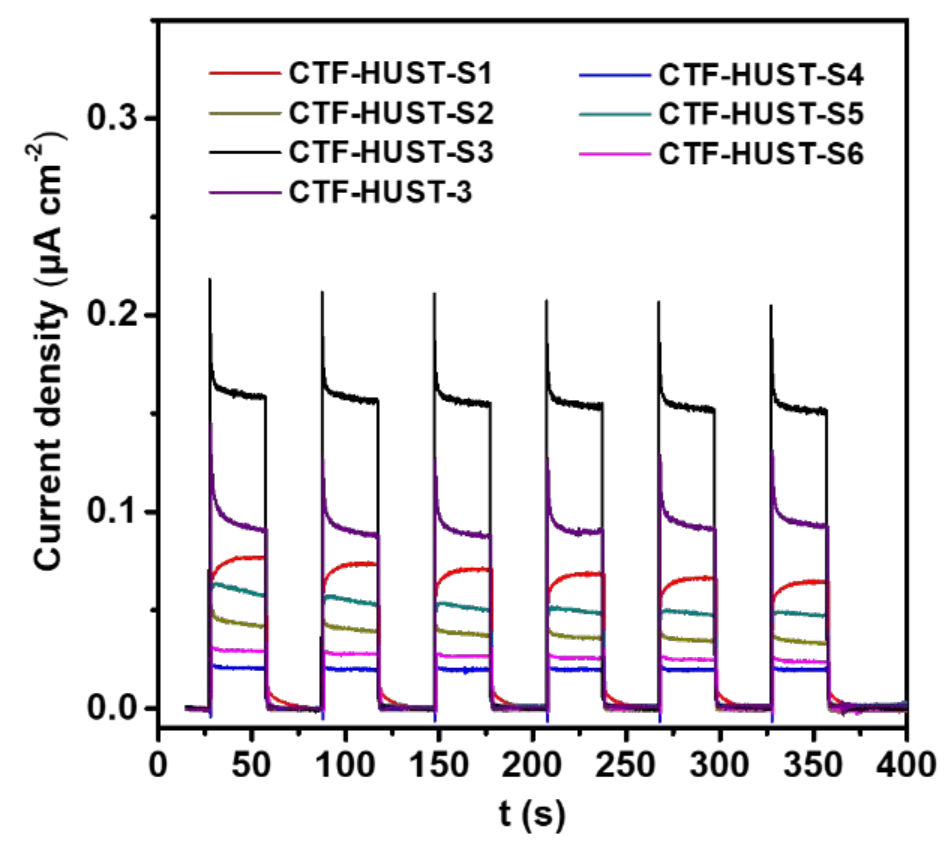

Figure S32. Time correlated transient photocurrent response of CTF-HUST-Sx

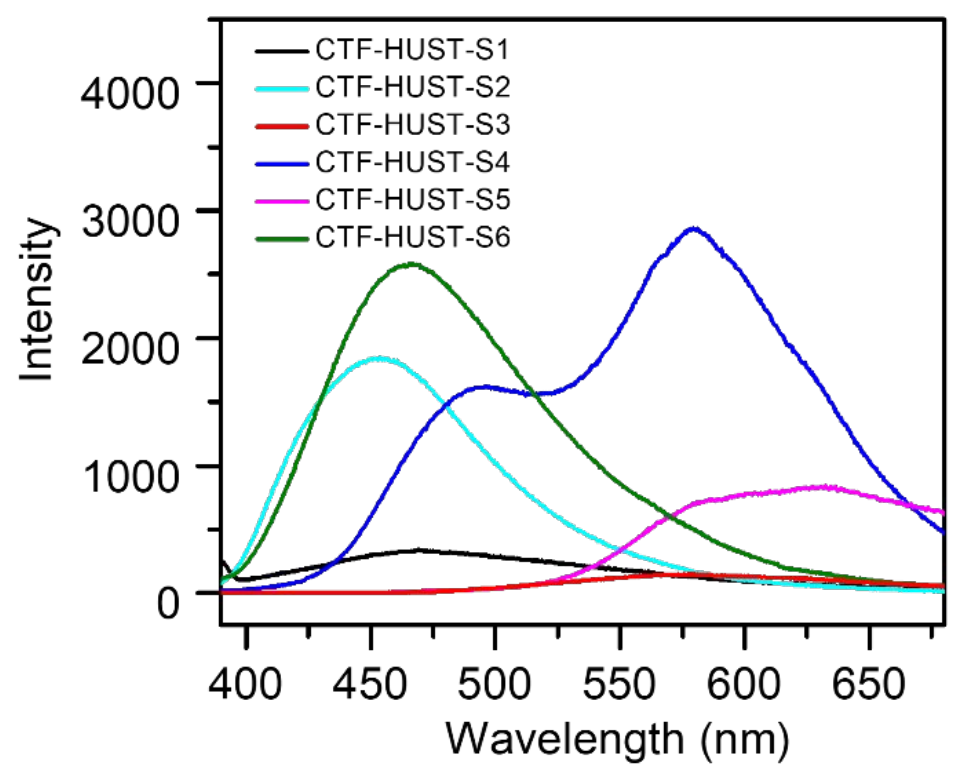

Figure S33. Steady-state photoluminescence spectra of CTF-HUST-Sx 
15. Photocatalytic hydrogen production performance of CTF-HUST-Sx

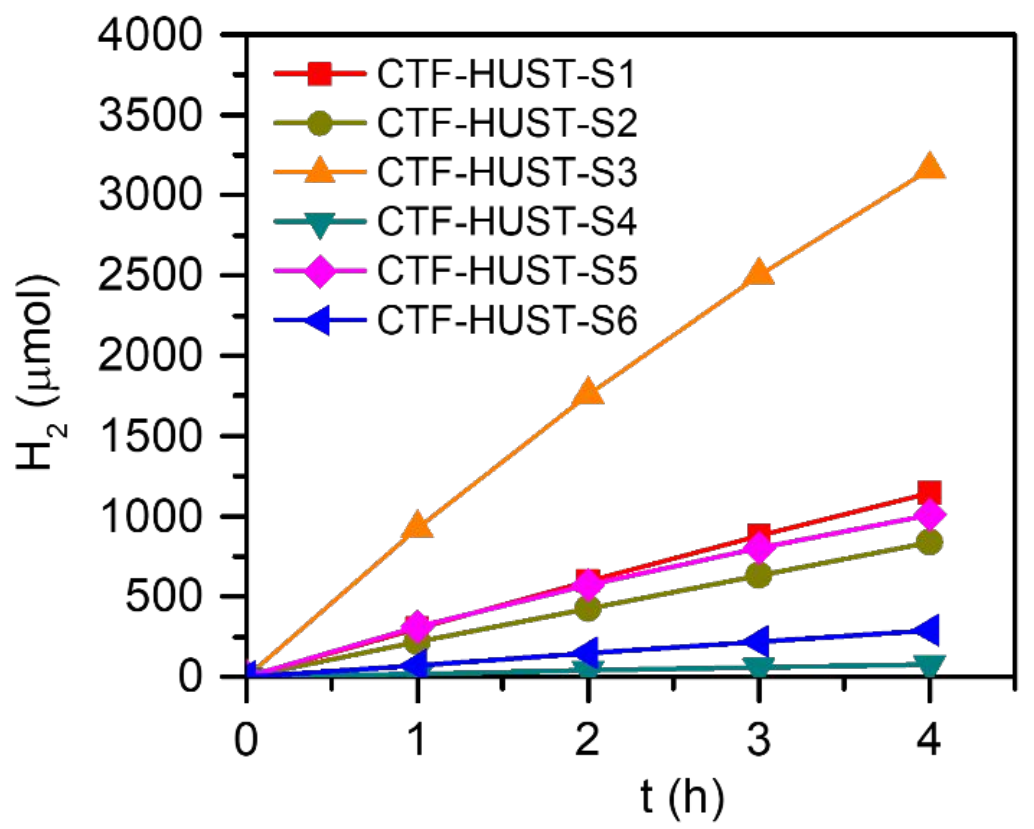

Figure S34. Time course of $\mathrm{H}_{2}$ production of CTFs from "sea water".

(a)

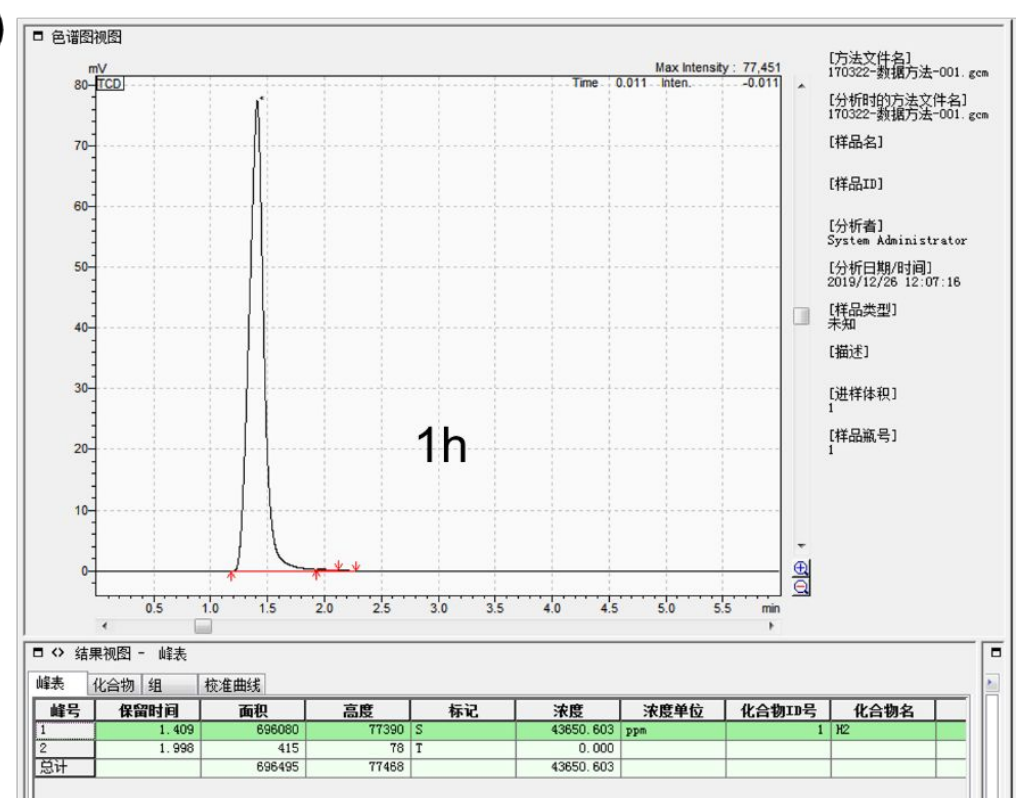


(b)

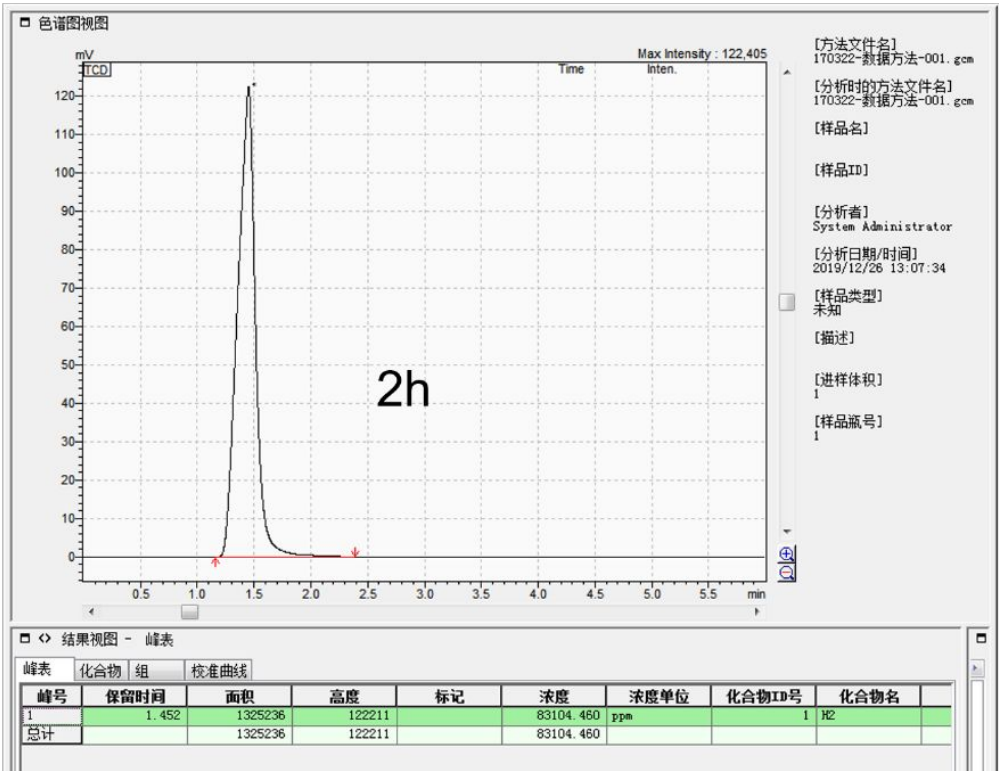

(c)

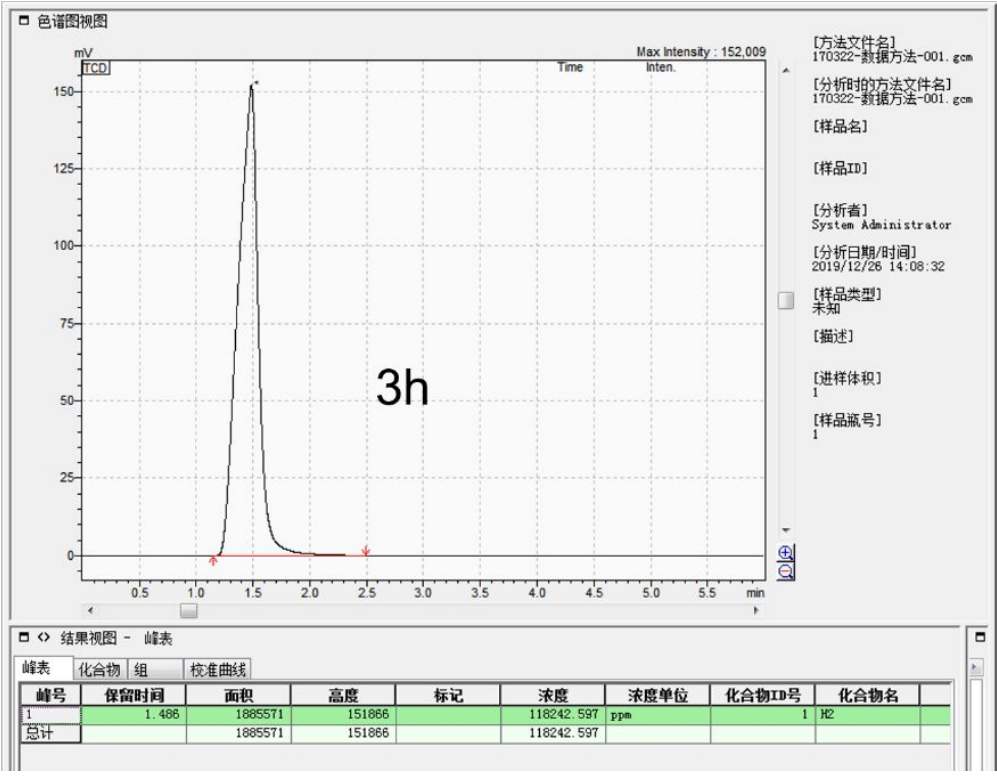

(d)

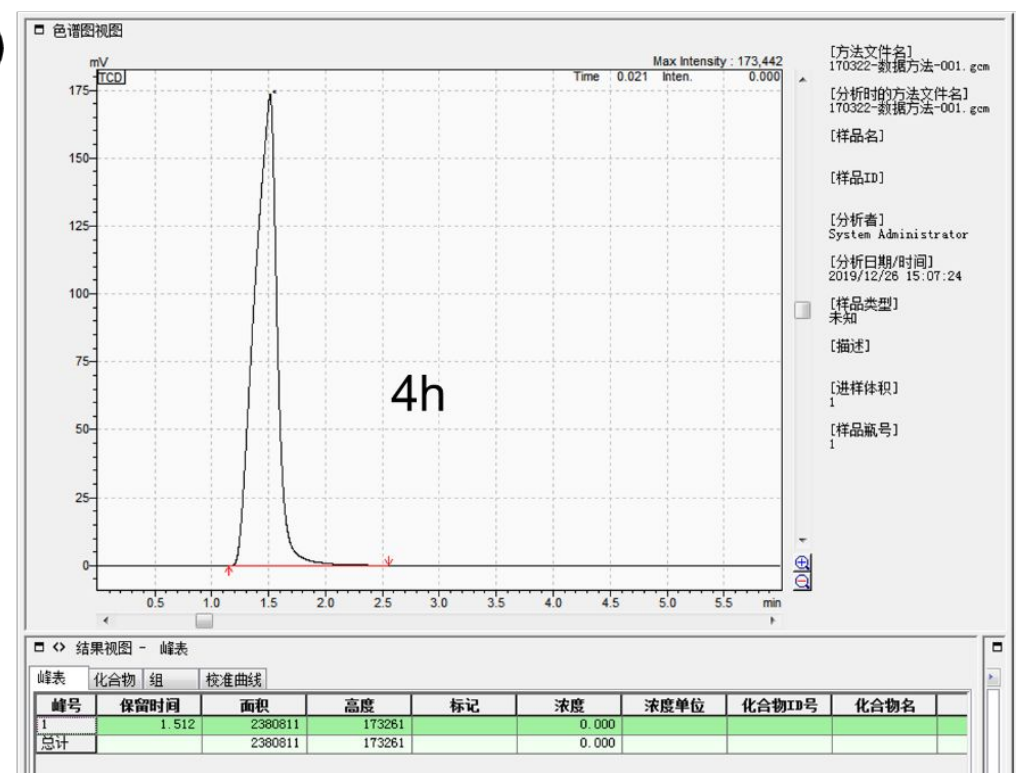


Figure S35. GC spectrum of the hydrogen evolution from "sea water" by CTF-HUST-S3, recorded at time interval of $1 \mathrm{~h}$.
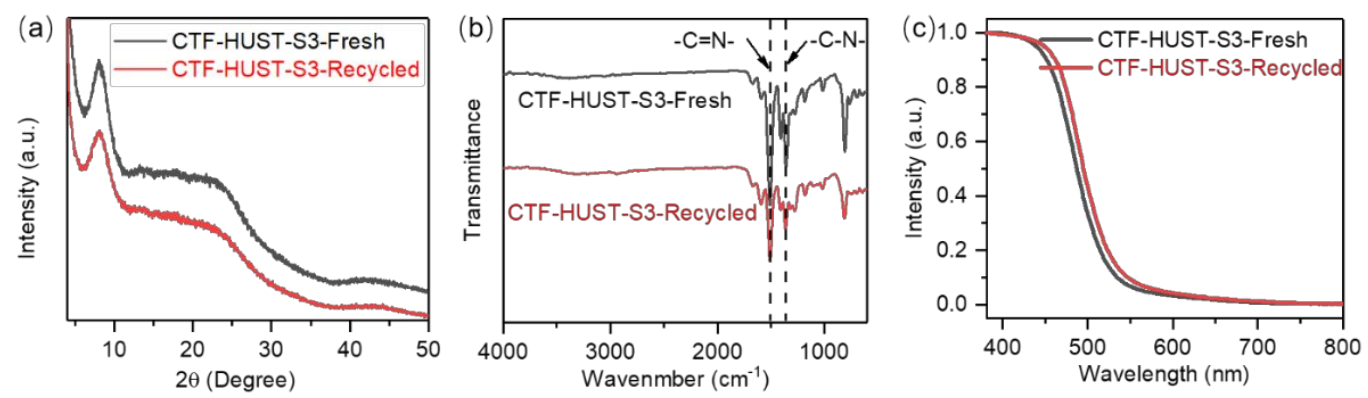

Figure S36. Structural characterization of CTF-HUST-S3 before and after photocatalysis: XRD results (a); FT-IR results (b) and UV-Vis absorption (c).

From the results of the XRD and FT-IR, the chemical structure and crystalline structure of the recycled CTF-HUST-S3 are kept well after five cycles in photocatalysis.

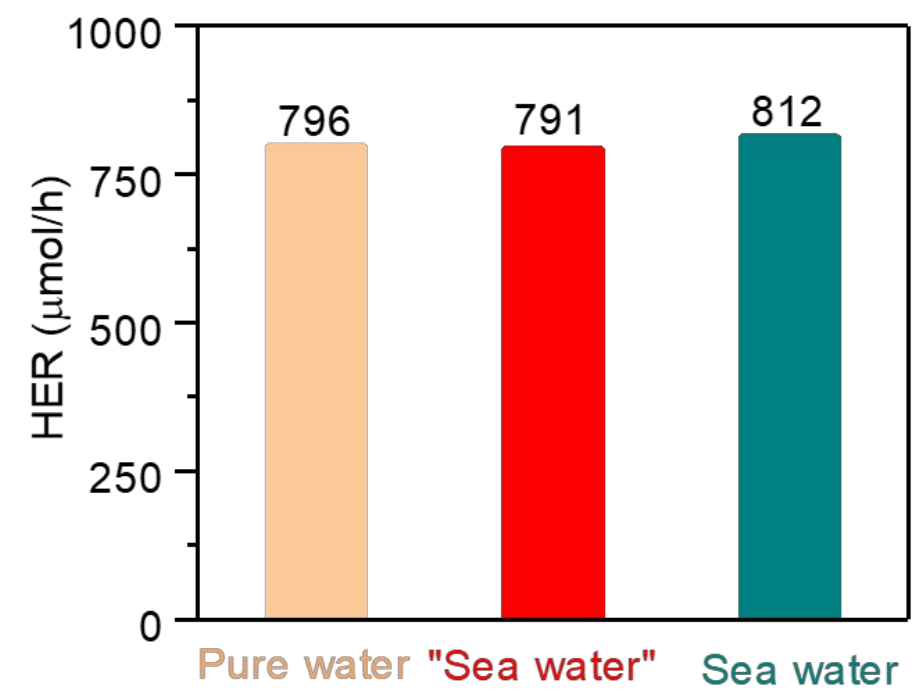

Figure S37. The photocatalytic hydrogen production of CTF-HUST-S3 in Pure water, "Sea water" and Sea water, respectively. (The sea water is from East China Sea near Xiamen, which was used in this experiment after filtration to remove the solid impurities) 


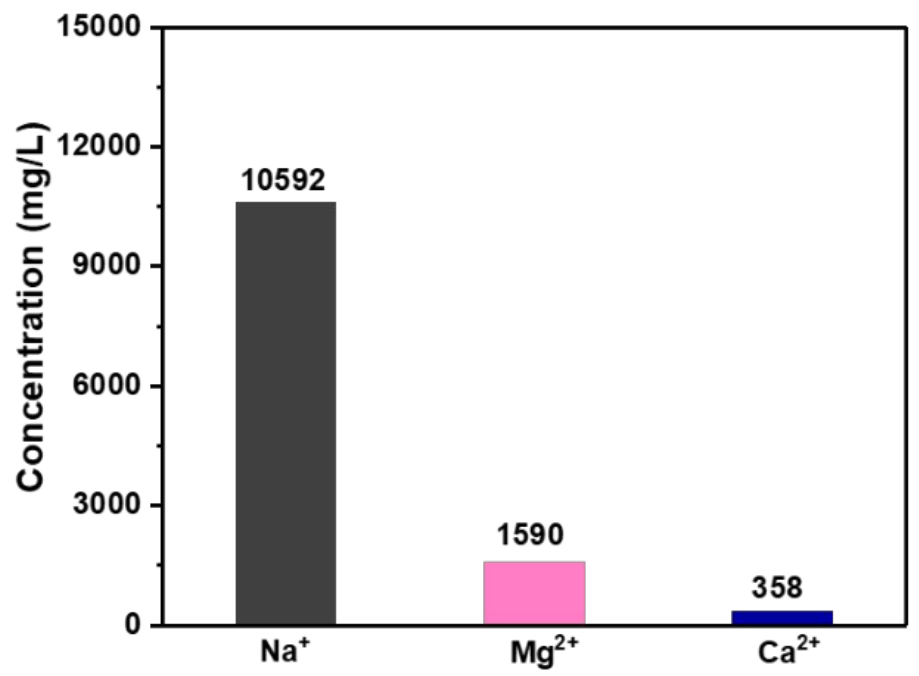

Figure S38. The cation concentration in sea water according to ICP testing. 


\section{Elemental analysis of CTF-HUST-Sx and CTF-HUST-2 and CTF-HUST-3}

Table S2. Summary of the elemental analysis results of CTF-HUST-Sx

\begin{tabular}{cccccccccc}
\hline \multirow{2}{*}{ Sample } & \multicolumn{2}{c}{$\mathrm{C}(\%)$} & \multicolumn{2}{c}{$\mathrm{N}(\%)$} & \multicolumn{2}{c}{$\mathrm{H}(\%)$} & \multicolumn{2}{c}{$\mathrm{S}(\%)$} \\
\cline { 2 - 9 } & Exp. & Cal. & Exp. & Cal. & Exp. & Cal. & Exp. & Cal. \\
\hline CTF-HUST-S1 & 69.34 & 74.99 & 19.55 & 21.86 & 3.967 & 3.15 & 0.357 & $/$ \\
CTF-HUST-S2 & 73.87 & 77.36 & 16.56 & 19.46 & 4.250 & 3.18 & 0.412 & $/$ \\
CTF-HUST-S3 & 70.46 & 76.69 & 17.69 & 19.87 & 4.051 & 3.43 & 0.515 & $/$ \\
CTF-HUST-S4 & 65.92 & 72.85 & 20.89 & 24.05 & 3.854 & 3.09 & 0.338 & $/$ \\
CTF-HUST-S5 & 58.32 & 64.30 & 16.87 & 17.91 & 2.954 & 2.42 & 10.263 & 15.37 \\
CTF-HUST-S6 & 72.05 & 79.99 & 16.01 & 16.46 & 4.205 & 3.53 & 0.203 & $/$ \\
\hline
\end{tabular}

Table S3. Comparison of the elemental analysis results of CTF-HUST-Sx and CTF-HUST-x

\begin{tabular}{lccccccccc}
\hline \multirow{2}{*}{ Sample } & \multicolumn{2}{c}{$\mathrm{C}(\%)$} & \multicolumn{2}{c}{$\mathrm{N}(\%)$} & \multicolumn{2}{c}{$\mathrm{H}(\%)$} & \multicolumn{2}{c}{$\mathrm{S}(\%)$} \\
\cline { 2 - 9 } & Exp. & Cal. & Exp. & Cal. & Exp. & Cal. & Exp. & Cal. \\
\hline CTF-HUST-S2 & 73.87 & & 16.56 & & 4.25 & & 0.412 & \\
CTF-HUST-2 & 67.60 & & 16.29 & & 4.55 & & 0.185 & \\
CTF-HUST-S3 & 70.46 & & 17.69 & & 4.05 & & 0.515 & \\
CTF-HUST-3 & 68.94 & & 13.39 & & 4.73 & & 0.558 & \\
\hline
\end{tabular}




\section{Surface area and porosity of CTF-HUST-Sx}

Table S4. Surface area and porosity of CTF-HUST-Sx

\begin{tabular}{lccccc}
\hline \multicolumn{1}{c}{ Sample } & $\begin{array}{c}\mathrm{S}_{\mathrm{BET}}{ }^{\mathrm{a}} \\
\left(\mathrm{m}^{2} \mathrm{~g}^{-1}\right)\end{array}$ & $\begin{array}{c}\mathrm{S}_{\mathrm{L}}{ }^{\mathrm{b}} \\
\left(\mathrm{m}^{2} \mathrm{~g}^{-1}\right)\end{array}$ & $\begin{array}{c}\text { M.A. } \\
\left(\mathrm{m}^{\mathrm{c}} \mathrm{g}^{-1}\right)\end{array}$ & $\begin{array}{c}\mathrm{PV}^{\mathrm{d}} \\
\left(\mathrm{cm}^{3} \mathrm{~g}^{-1}\right)\end{array}$ & $\begin{array}{c}\mathrm{MPV}^{\mathrm{e}} \\
\left(\mathrm{cm}^{3} \mathrm{~g}^{-1}\right)\end{array}$ \\
\hline CTF-HUST-S1 & 617 & 1432 & 338 & 0.93 & 0.17 \\
CTF-HUST-S2 & 623 & 1236 & 408 & 0.71 & 0.22 \\
CTF-HUST-S3 & 715 & 2072 & 281 & 1.28 & 0.15 \\
CTF-HUST-S4 & 470 & 926 & 301 & 0.43 & 0.16 \\
CTF-HUST-S5 & 454 & 1018 & 213 & 0.57 & 0.11 \\
CTF-HUST-S6 & 800 & 2032 & 361 & 1.16 & 0.19 \\
\hline
\end{tabular}

${ }^{\text {a }}$ Surface area calculated from nitrogen adsorption isotherms at $77.3 \mathrm{~K}$ using BET equation.

${ }^{\mathrm{b}}$ Surface area calculated from nitrogen adsorption isotherms at $77.3 \mathrm{~K}$ using Langmuir equation.

${ }^{\mathrm{c}}$ t-Plot micropore area.

${ }^{\mathrm{d}}$ Pore volume calculated from nitrogen isotherm at $\mathrm{P} / \mathrm{P}_{0}=0.995,77.3 \mathrm{~K} .{ }^{\mathrm{e}}$ t-Plot micropore volume. 
18. Comparison of the photocatalytic performance of CTF-HUST-Sx samples with reported results

Table S5. Comparison of the photocatalytic performance with other reported results

\begin{tabular}{|c|c|c|c|c|c|}
\hline Catalyst & Condition & $\begin{array}{c}\text { Amount } \\
\text { of catalyst } \\
(\mathrm{mg}) \\
\end{array}$ & $\begin{array}{c}\text { Hydrogen } \\
\text { evolution rate } \\
\left.(\mu \mathrm{mol} \mathrm{h})^{-1}\right) \\
\end{array}$ & $\begin{array}{l}\text { AQY } \\
(\%)\end{array}$ & Ref. \\
\hline CTF-C1 & $\begin{array}{c}>420 \mathrm{~nm} ; \\
\text { TEOA } \\
3 \mathrm{wt} \% \mathrm{Pt}\end{array}$ & 50 & 255 & I & [2] \\
\hline CTF-HUST-A1 & $\begin{array}{c}>420 \mathrm{~nm} ; \\
\text { TEOA } \\
3 \mathrm{wt} \% \mathrm{Pt}\end{array}$ & 50 & 460 & $\begin{array}{c}7.4 \% \\
(420 \mathrm{~nm})\end{array}$ & [3] \\
\hline $\mathrm{N}_{3}-\mathrm{COF}$ & $\begin{array}{c}>420 \mathrm{~nm} \\
\text { TEOA } \\
3 \mathrm{wt} \% \mathrm{Pt}\end{array}$ & 5 & 9 & $\begin{array}{c}0.45 \% \\
(450 \mathrm{~nm})\end{array}$ & [4] \\
\hline $\mathrm{N}_{2}-\mathrm{COF}$ & $\begin{array}{c}\text { AM } 1.5 \mathrm{G} \\
\text { TEOA } \\
8 \mathrm{wt} \% \mathrm{Co}-1 \\
\end{array}$ & 5 & 3.91 & $\begin{array}{c}0.16 \% \\
(400 \mathrm{~nm})\end{array}$ & [5] \\
\hline TFPT-COF & $\begin{array}{c}>420 \mathrm{~nm} ; \\
\text { TEOA }\end{array}$ & 10 & 20 & $2.2 \%$ & [6] \\
\hline TP-BDDA-COF & $\begin{array}{c}>395 \mathrm{~nm} \\
\text { TEOA } \\
3 \mathrm{wt} \% \mathrm{Pt} \\
\end{array}$ & 10 & 3.24 & $\begin{array}{c}1.8 \% \\
(520 \mathrm{~nm})\end{array}$ & [7] \\
\hline FS-COF-WS5F & $\begin{array}{c}>420 \mathrm{~nm}, \\
\text { ascorbic acid, } \\
0.038 \mathrm{wt} \% \mathrm{Pt},\end{array}$ & 5 & 81.5 & $\begin{array}{c}2.2 \% \\
(600 \mathrm{~nm})\end{array}$ & [8] \\
\hline $\begin{array}{c}\mathrm{NH}_{2}-\mathrm{UiO}-66 / \\
\mathrm{TpPa}-1-\mathrm{COF}\end{array}$ & $\begin{array}{l}>420 \mathrm{~nm} \\
\text { sodium } \\
\text { ascorbate }\end{array}$ & 10 & 234.1 & / & [9] \\
\hline sp2C-COF ${ }_{\text {ERDN }}$ & $\begin{array}{c}>420 \mathrm{~nm} \\
\mathrm{TEOA} \\
3 \% \mathrm{Pt} \\
\end{array}$ & 50 & 106 & $\begin{array}{c}0.46 \% \\
(420 \mathrm{~nm})\end{array}$ & {$[10]$} \\
\hline Pt-PVP-TP-COF & $\begin{array}{c}>420 \mathrm{~nm} \\
\text { TEOA } \\
6 \% \mathrm{Pt}-\mathrm{PVP} \\
\end{array}$ & 10 & 84.2 & $\begin{array}{c}0.4 \% \\
(475 \mathrm{~nm})\end{array}$ & [11] \\
\hline CTF-HUST-S3 & $\begin{array}{c}>420 \mathrm{~nm} \\
\text { TEOA } \\
3 \% \mathrm{Pt}\end{array}$ & 50 & 791 & $\begin{array}{c}12.78 \% \\
(420 \mathrm{~nm}) \\
11.44 \\
(500 \mathrm{~nm})\end{array}$ & $\begin{array}{l}\text { This } \\
\text { work }\end{array}$ \\
\hline
\end{tabular}




\section{Computational details}

Periodic DFT calculations on the CTF crystal structures were carried out within the plane-wave pseudopotential formalism, using the Vienna ab initio Simulation Package (VASP) code. ${ }^{[12]}$ A kinetic-energy cutoff of $500 \mathrm{eV}$ was used to define the plane-wave basis set, and the electronic Brillouin zone was integrated using $\Gamma$-centred Monkhorst-Pack grids with the smallest allowed spacing between k-points (KSPACING) being $0.2 \AA^{-1}$ for all the CTF structures. Geometry optimizations, involving both lattice parameters and atomic positions, were performed employing the Perdew-Burke-Ernzerhof exchange-correlation functional with the DFTD3(BJ) dispersion correction. The electronic structure of each optimized CTF structure was then computed using a screened hybrid exchange-correlation functional (HSE06), giving key electronic properties, such as band gap and electrostatic potential, for comparison of the CTFs. Tolerances of $10^{-6} \mathrm{eV}$ and $10^{-2} \mathrm{eV} \AA^{-1}$ were applied during the optimization of the Kohn-Sham wavefunctions and the geometry optimizations, respectively. Within periodic boundary conditions, the electronic eigenvalues are given with respect to an internal reference. In order to achieve valence band alignment, using a common vacuum level, so that band energies can be compared for the different CTF structures, we followed the well-established 'slab' approach — a slab comprising 5 adjacent CTF layers, surrounded by a vacuum layer of $25 \AA$ on both sides - to determine the vacuum level, based on the electrostatic potential at the centre of the vacuum layer. Furthermore, the effects of solvation by water on the slab surfaces were modelled by using VASPsol. ${ }^{[13,14]}$

Periodic band structure calculations take into account the influence of layer stacking and, by necessity, approximate the IP and EA by the Kohn-Sham valence band maximum (VBM) and conduction band minimum (CBM), respectively. However, calculations of the excited states of periodic crystal structures are computationally difficult and demanding. By contrast, cluster calculations, although limited to a fragment, allow us to calculate the exciton potentials and to analyse in detail the electronic and excited-state properties of the system. The former is particularly important for polymeric photocatalysts, where the exciton binding energy is generally large relative to $k \mathrm{~T}$ (26 meV at room temperature), such that spontaneous dissociation of excitons into free electrons and holes is difficult. 
Representative molecular fragments-CTF-HUST-S1(L) to S6(L) of CTF-HUST-S1 to S6, respectively_-were calculated for their standard reduction potentials of half-reactions for free electrons/holes and excitons, using density functional theory (DFT) and time-dependent DFT (TD-DFT). The wB97XD density functional was used for all the DFT and TD-DFT calculations, together with the Def2-SVP basis set, using the Gaussian 16 software. ${ }^{[15]}$ The effect of solvation by water was accounted for by using the PCM/SMD solvation model. Vertical reduction potentials (i.e., IP and EA) and vertical exciton potentials (i.e., IP* and EA*) were calculated using the geometry optimized in the ground state, confirmed to be a true minimum by a frequency calculation. All electron excitation analyses were performed using Multiwfn. ${ }^{[16]}$

Table S6. PBE-D3(BJ) simulated cell parameters for AA-stacked models of CTF-HUST-S1 to S6.

\begin{tabular}{|c|c|c|c|c|c|c|c|}
\hline & Space group & $\mathrm{a}(\AA)$ & $\mathrm{b}(\AA)$ & $\mathrm{c}(\AA)$ & $\alpha\left(^{\circ}\right)$ & $\beta\left(^{\circ}\right)$ & $\gamma\left({ }^{\circ}\right)$ \\
\hline $\begin{array}{c}\text { CTF-HUST- } \\
\text { S1 }\end{array}$ & $\mathrm{P} 6 / \mathrm{mmm}$ & 14.5352 & 14.5352 & 3.6052 & 90.0 & 90.0 & 120.0 \\
\hline $\begin{array}{c}\text { CTF-HUST- } \\
\text { S2 }\end{array}$ & $\mathrm{P} 6 / \mathrm{mmm}$ & 29.5202 & 29.5202 & 3.6160 & 90.0 & 90.0 & 120.0 \\
\hline $\begin{array}{c}\text { CTF-HUST- } \\
\text { S3 }\end{array}$ & $\mathrm{P} 1$ & 26.6089 & 26.6369 & 3.7922 & 82.1 & 79.8 & 120.3 \\
\hline $\begin{array}{c}\text { CTF-HUST- } \\
\text { S4 }\end{array}$ & $\mathrm{P} 6 / \mathrm{m}$ & 29.3213 & 29.3213 & 3.6181 & 90.0 & 90.0 & 120.0 \\
\hline $\begin{array}{c}\text { CTF-HUST- } \\
\text { S5 }\end{array}$ & $\mathrm{P} 6 / \mathrm{m}$ & 26.8542 & 26.8542 & 3.6611 & 90.0 & 90.0 & 120.0 \\
\hline $\begin{array}{c}\text { CTF-HUST- } \\
\text { S6 }\end{array}$ & $\mathrm{P} 6 / \mathrm{mmm}$ & 29.3053 & 29.3053 & 3.6162 & 90.0 & 90.0 & 120.0 \\
\hline
\end{tabular}


Table S7. Calculated electronic and excited-state properties for the representative molecular fragments S1(L) to S6(L) of CTF-HUST-S1 to S6, respectively.

\begin{tabular}{ccccccc}
\hline CTF-HUST-Sx & S1(L) & S2(L) & S3(L) & S4(L) & S5(L) & S6(L) \\
\hline IP & 2.650 & 2.128 & 1.068 & 2.445 & 1.978 & 2.058 \\
EA & -1.779 & -1.884 & -1.902 & -1.789 & -1.685 & -1.888 \\
EA* & 2.505 & 2.215 & 1.690 & 2.251 & 1.950 & 2.368 \\
IP* & -1.634 & -1.971 & -2.523 & -1.595 & -1.657 & -2.198 \\
$E_{\text {gap }}$ & 4.284 & 4.099 & 3.591 & 4.040 & 3.635 & 4.256 \\
$\Delta D$ & 0.000 & 0.016 & 2.651 & 0.000 & 0.002 & 1.266 \\
$S r$ & 0.811 & 0.814 & 0.691 & 0.792 & 0.801 & 0.819 \\
$\Delta \sigma$ & 0.446 & 0.855 & 1.243 & 0.601 & 0.818 & 0.816 \\
$E_{\mathrm{sol}}$ & -0.021 & -0.020 & -0.020 & -0.021 & -0.021 & -0.020 \\
$\Delta E_{\mathrm{S} 1 \rightarrow \mathrm{T} 1}$ & 1.462 & 1.305 & 0.856 & 1.278 & 1.315 & 1.465 \\
$\Delta E_{\mathrm{T} 2 \rightarrow \mathrm{T} 1}$ & 0.080 & 0.099 & 0.008 & 0.130 & 0.607 & 0.086 \\
\hline
\end{tabular}

IP: ionization potential, EA: electron affinity; IP* and EA* are the corresponding exciton potentials. $E_{\text {gap }}$ : optical gap, calculated as the energy difference between the S1 and S0 states.

$\Delta D$ : difference between the excited-state and the ground-state dipole moments of the molecule, a measure of the extent of charge redistribution between the two states.

$S r$ index quantify the overlap between the hole distribution, $\rho^{\text {hole }}(\boldsymbol{r})$, and the electron distribution, $\rho^{\text {electron }}(\boldsymbol{r}) . S r$ varies between 0 (no overlap) and 1 (complete overlap); the larger the value is, the greater the extent of overlap is.

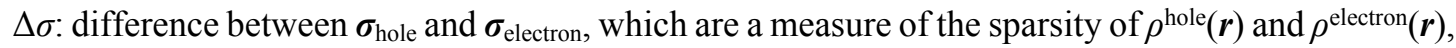
respectively.

$E_{\text {sol }}$ : solvation energy of the molecule in water, approximated as the energy difference between the molecule in solvation and in vacuum, divided by the number of non-hydrogen atoms in the molecule. $\Delta E_{\mathrm{S} 1 \rightarrow \mathrm{T} 1}$ : energy difference between the first singlet state (S1) and the first triplet state (T1). The smaller the $\Delta E_{\mathrm{S} 1 \rightarrow \mathrm{T} 1}$ value, the larger the spin-orbital coupling, and ultimately the more probable and faster the intersystem crossing.

$\Delta E_{\mathrm{T} 2 \rightarrow \mathrm{T} 1}$ : energy difference between the second triplet state (T2) and the first triplet state (T1).

Our calculations (Table S6) predict, for CTF-HUST-S3(L), that the first triplet (T1) state lies below the first singlet state by only $0.856 \mathrm{eV}$ and that the $\mathrm{T} 2$ state is degenerate (above $\mathrm{T} 1$ by only $0.008 \mathrm{eV}$ ). This $\Delta E_{\mathrm{S} 1 \rightarrow \mathrm{T} 1}$ value is on a par with experimentally measured counterparts for many semiconducting conjugated polymers that show intersystem crossing ${ }^{[17]}$ suggesting the possibility of formation of triplet excitons in CTF-HUST-S3. Triplet excitons, in general, have the advantage of a much longer lifetime, and thereby a longer diffusion length, than the singlet excitons. This is beneficial for singlet excitons in the bulk to diffuse and dissociate at the photocatalyst-solution interface, which would otherwise undergo recombination to the ground 
state. This hypothesized intersystem crossing of singlet excitons to triplet excitons in CTFHUST-S3 might have contributed to its lowest photoluminescence intensity among the series (Figure S28), because intersystem crossing competes with radiative decay of photoexcitation by photoluminescence. However, time-resolved spectroscopy measurements, such as femtosecond UV-vis transient absorption spectroscopy, will be required to fully probe and understand such photoinduced processes in the excited electronic states, which is beyond the scope of the current work but will form an in-depth, follow-up study. 
Table S8. Calculated TD-DFT (TD-wB97XD) excitation energies $(E)$ for the lowest three transitions (S1-S3), with corresponding oscillator strengths $(f)$, of the representative molecular fragments S1(L) to S6(L) of CTF-HUST-S1 to S6, respectively. For each excited state, with $f>$ 0.001 , the $S r$ index was calculated to quantify the overlap between the hole distribution, $\rho^{\text {hole }}(\boldsymbol{r})$, and the electron distribution, $\rho^{\text {electron }}(\boldsymbol{r})$, together with the distance $(D)$ between the centroid of $\rho^{\text {hole }}(\boldsymbol{r})$ and the centroid of. $\rho^{\text {electron }}(\boldsymbol{r}) . S r$ varies between 0 (no overlap) and 1 (complete overlap); the larger the value is, the greater the extent of overlap is.

\begin{tabular}{cccccc}
\hline $\begin{array}{c}\text { CTF-HUST- } \\
\text { Sx }\end{array}$ & State & $E(\mathrm{eV}, \mathrm{nm})$ & $f$ & $S r$ (a.u. $)$ & $D(\AA)$ \\
\hline S1(L) & S1 & $4.2840(289.41)$ & 2.404 & 0.811 & 0.000 \\
& S2 & $4.3735(283.49)$ & 0.000 & & \\
& S3 & $4.5659(271.54)$ & 0.000 & & \\
\hline S2(L) & S1 & $4.0993(302.45)$ & 2.828 & 0.814 & 0.009 \\
& S2 & $4.3632(284.16)$ & 0.011 & 0.465 & 0.668 \\
& S3 & $4.3643(284.09)$ & 0.007 & 0.455 & 0.406 \\
\hline S3(L) & S1 & $3.5913(345.23)$ & 1.460 & 0.691 & 1.415 \\
& S2 & $3.5995(344.45)$ & 1.386 & 0.675 & 1.572 \\
& S3 & $4.2657(290.65)$ & 0.040 & 0.656 & 0.848 \\
\hline S4(L) & S1 & $4.0400(306.89)$ & 2.627 & 0.792 & 0.000 \\
& S2 & $4.3719(283.59)$ & 0.000 & & \\
& S3 & $4.3780(283.20)$ & 3.458 & 0.803 & 0.000 \\
\hline S5(L) & S1 & $3.6346(341.12)$ & 2.040 & 0.801 & 0.001 \\
& S2 & $4.0949(302.78)$ & 0.011 & 0.686 & 0.003 \\
& S3 & $4.3752(283.38)$ & 3.420 & 0.806 & 0.000 \\
\hline S6(L) & S1 & $4.2558(291.33)$ & 2.730 & 0.819 & 0.668 \\
& S2 & $4.2602(219.03)$ & 2.752 & 0.816 & 0.619 \\
& S3 & $4.3876(282.58)$ & 1.758 & 0.812 & 0.129 \\
\hline
\end{tabular}




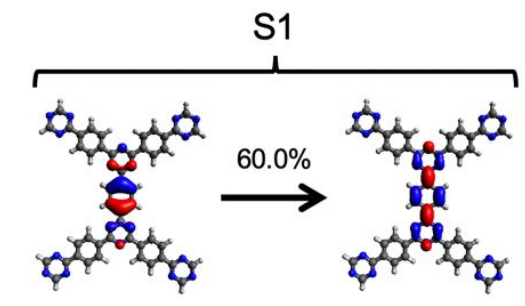

Figure S39. Natural transition orbitals (NTOs) for the major component transition(s) of each of the first three excited state (S1-S3) of CTF-HUST-S1(L), with the corresponding contribution from the NTO pair labelled on the arrow indicating the transition. Results for S2 and S3 are omitted here because their oscillator strengths are smaller than 0.001 .
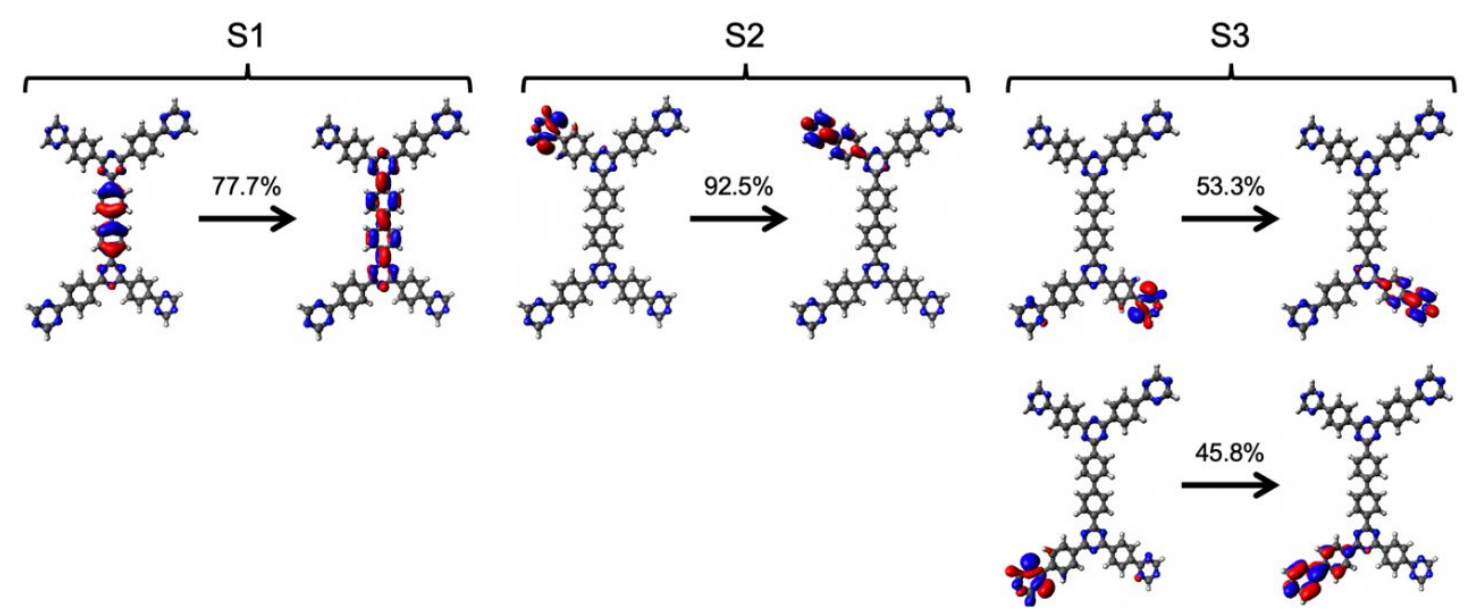

Figure S40. Natural transition orbitals (NTOs) for the major component transition(s) of each of the first three excited state (S1-S3) of CTF-HUST-S2(L), with the corresponding contribution from the NTO pair labelled on the arrow indicating the transition. 

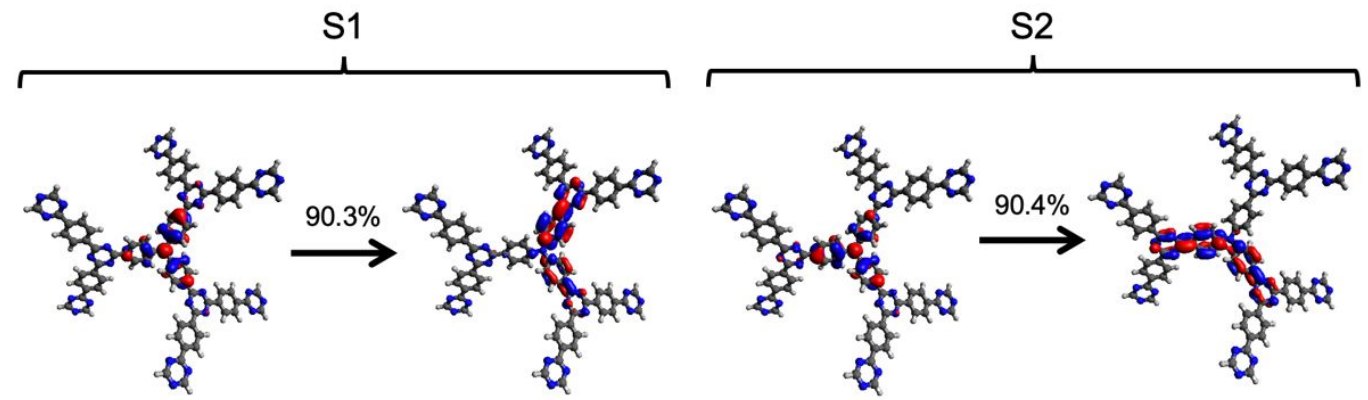

S3

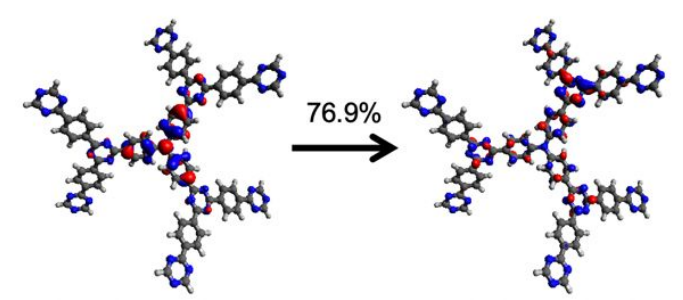

Figure S41. Natural transition orbitals (NTOs) for the major component transition(s) of each of the first three excited state (S1-S3) of CTF-HUST-S3(L), with the corresponding contribution from the NTO pair labelled on the arrow indicating the transition.
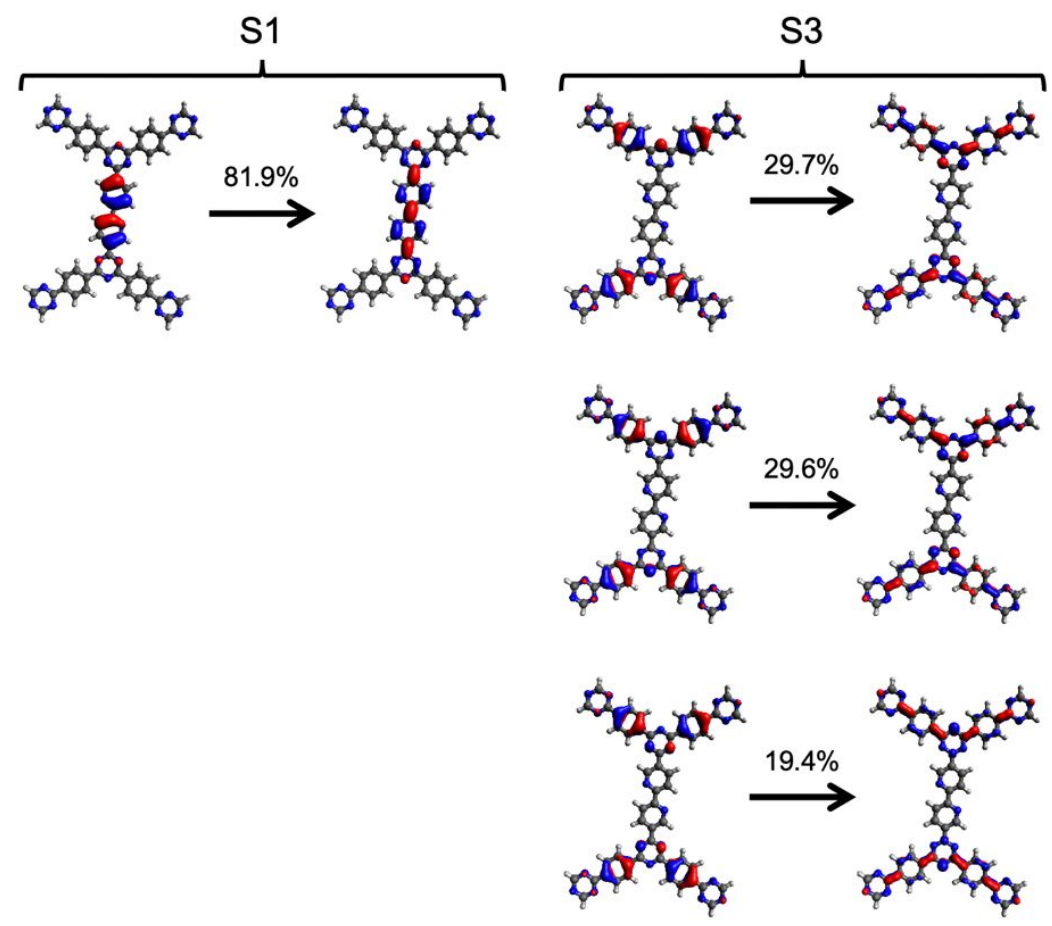

Figure S42. Natural transition orbitals (NTOs) for the major component transition(s) of each of the first three excited state (S1-S3) of CTF-HUST-S4(L), with the corresponding contribution from the NTO pair labelled on the arrow indicating the transition. Results for S2 are omitted here because its oscillator strength is smaller than 0.001 . 
S1

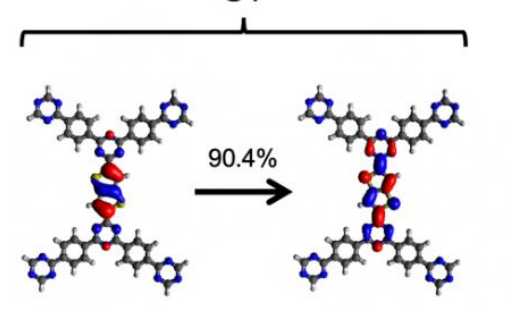

S2

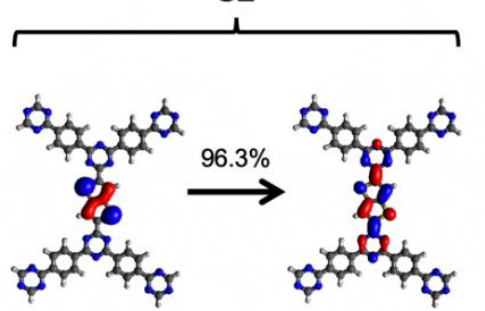

S3
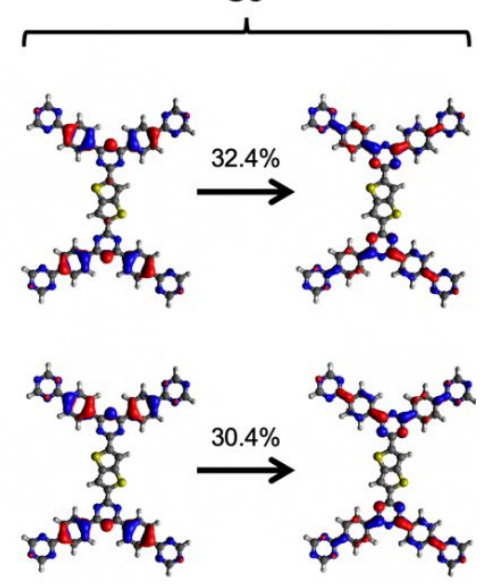

Figure S43. Natural transition orbitals (NTOs) for the major component transition(s) of each of the first three excited state (S1-S3) of CTF-HUST-S5(L), with the corresponding contribution from the NTO pair labelled on the arrow indicating the transition.

S1

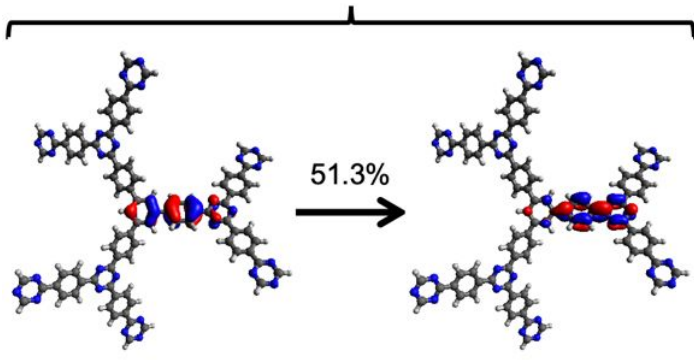

S3

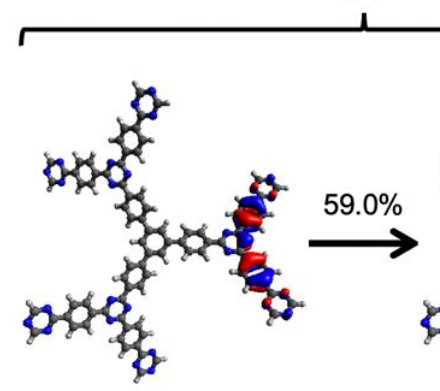

S2
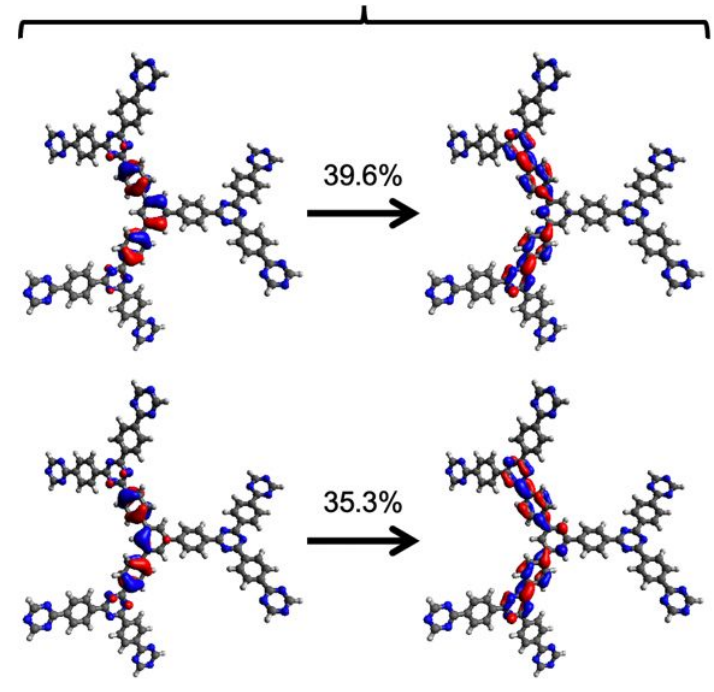

Figure S44. Natural transition orbitals (NTOs) for the major component transition(s) of each of the first three excited state (S1-S3) of CTF-HUST-S6(L), with the corresponding contribution from the NTO pair labelled on the arrow indicating the transition. 


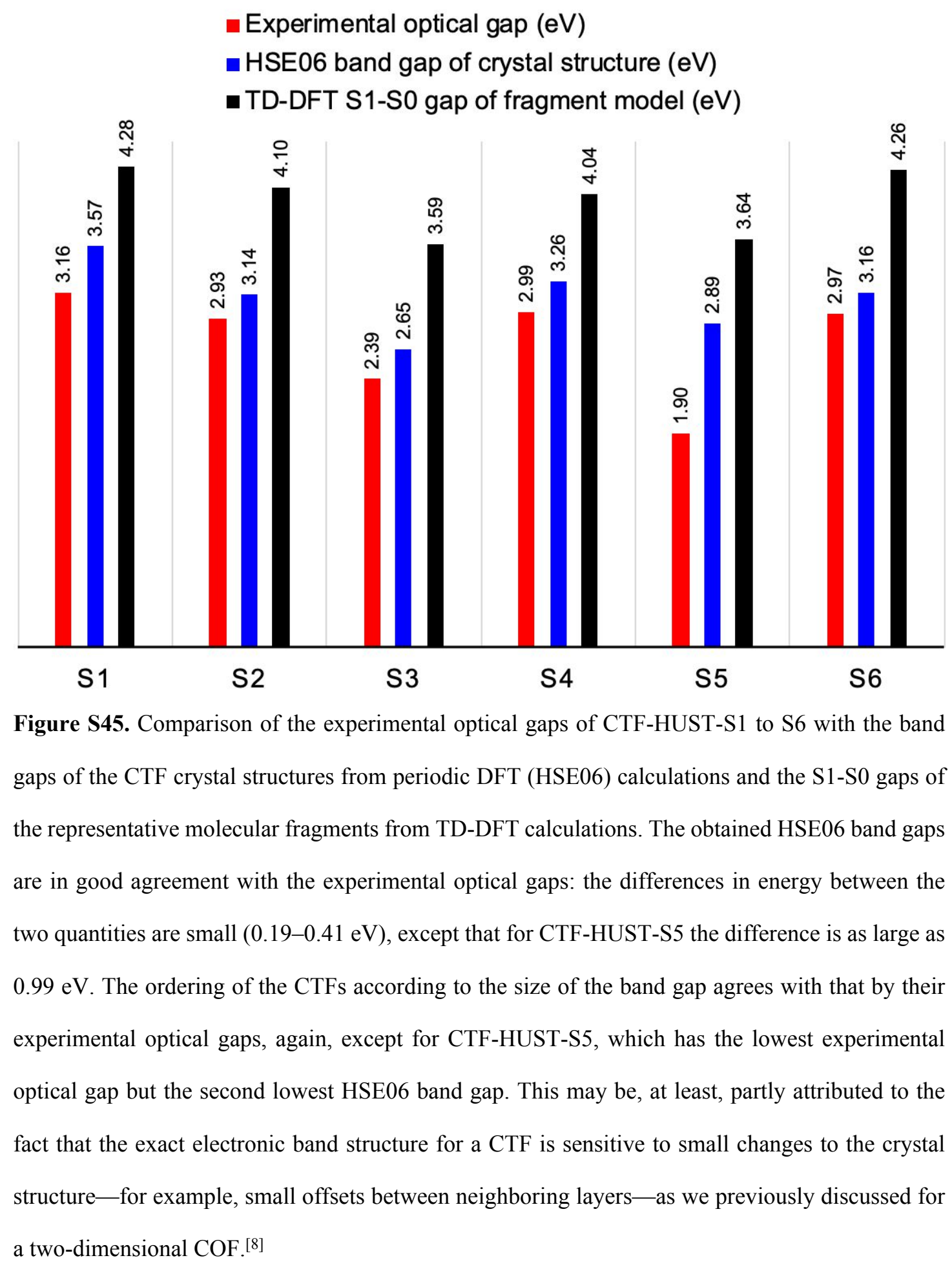




\section{References}

[1] K. Wang, L. Yang, X. Wang, L. Guo, G. Cheng, C. Zhang, S. Jin, B. Tan, A. Cooper, Angew. Chem. Int. Ed. 2017, 56, 14149.

[2] M. Liu, Q. Huang, S. Wang, Z. Li, B. Li, S. Jin, B. Tan, Angew. Chem. Int. Ed. 2018, 57, 11968.

[3] S. Zhang, G. Cheng, L. Guo, N. Wang, B. Tan, S. Jin, Angew. Chem. Int. Ed. 2020, 59, 6007.

[4] V. S. Vyas, F. Haase, L. Stegbauer, G. Savasci, F. Podjaski, C. Ochsenfeld, B. V. Lotsch, Nat. Commun. 2015, 6, 8508.

[5] T. Banerjee, F. Haase, G. Savasci, K. Gottschling, C. Ochsenfeld, B. V. Lotsch, J. Am. Chem. Soc. 2017, 139, 16228.

[6] L. Stegbauer, K. Schwinghammer, B. V. Lotsch, Chem. Sci. 2014, 5, 2789.

[7] P. Pachfule, A. Acharjya, J. Roeser, T. Langenhahn, M. Schwarze, R. Schomacker, A. Thomas, J. Schmidt, J. Am. Chem. Soc. 2018, 140, 1423.

[8] X. Wang, L. Chen, S. Y. Chong, M. A. Little, Y. Wu, W. H. Zhu, R. Clowes, Y. Yan, M. A. Zwijnenburg, R. S. Sprick, A. I. Cooper, Nat. Chem. 2018, 10, 1180.

[9] F. M. Zhang, J. L. Sheng, Z. D. Yang, X. J. Sun, H. L. Tang, M. Lu, H. Dong, F. C. Shen, J. Liu, Y. Q. Lan, Angew. Chem. Int. Ed. 2018, 57, 12106.

[10] E. Jin, Z. Lan, Q. Jiang, K. Geng, G. Li, X. Wang, D. Jiang, Chem 2019, 5, 1632.

[11] J. Ming, A. Liu, J. Zhao, P. Zhang, H. Huang, H. Lin, Z. Xu, X. Zhang, X. Wang, J. Hofkens, M. B. J. Roeffaers, J. Long, Angew. Chem. Int. Ed. 2019, 58, $18290 .$.

[12] Kresse, G. \& Furthmüller, J. Phys. Rev. B 1996, 54, 11169.

[13] K. Mathew, R. Sundararaman, K. Letchworth-Weaver, T. A. Arias, and R. G. Hennig, J. Chem. Phys. 2014, 140, 084106.

[14] M. Fishman, H. L. Zhuang, K. Mathew, W. Dirschka, and R. G. Hennig, Phys. Rev. B 2013, 87, 245402.

[15] M. J. Frisch, et al. Gaussian 16 revision A.03 (Gaussian, 2016).

[16] T. Lu, F. Chen, J. Comput. Chem. 2012, 33, 580.

[17] A. Köhler, D. Beljonne, Adv. Funct. Mater. 2004, 14, 11. 